University of Louisville

ThinkIR: The University of Louisville's Institutional Repository

Electronic Theses and Dissertations

8-2017

\title{
Residuated maps, the way-below relation, and contractions on probabilistic metric spaces.
}

M. Ryan Luke

University of Louisville

Follow this and additional works at: https://ir.library.louisville.edu/etd

Part of the Algebra Commons, Analysis Commons, and the Other Mathematics Commons

\section{Recommended Citation}

Luke, M. Ryan, "Residuated maps, the way-below relation, and contractions on probabilistic metric spaces." (2017). Electronic Theses and Dissertations. Paper 2746.

https://doi.org/10.18297/etd/2746

This Doctoral Dissertation is brought to you for free and open access by ThinkIR: The University of Louisville's Institutional Repository. It has been accepted for inclusion in Electronic Theses and Dissertations by an authorized administrator of ThinkIR: The University of Louisville's Institutional Repository. This title appears here courtesy of the author, who has retained all other copyrights. For more information, please contact thinkir@louisville.edu. 


\title{
RESIDUATED MAPS, THE WAY-BELOW RELATION, AND CONTRACTIONS ON PROBABILISTIC METRIC SPACES
}

\author{
By \\ M. Ryan Luke \\ B.S., Mississippi College, 2002 \\ M.S. Mississippi College, 2011 \\ M.A., University of Louisville, 2013
}

\begin{abstract}
A Dissertation
Submitted to the Faculty of the

College of Arts and Sciences of the University of Louisville in Partial Fulfillment of the Requirements

for the Degree of
\end{abstract}

Doctor of Philosophy

in

Applied and Industrial Mathematics

Department of Mathematics

University of Louisville

Louisville, KY

August 2017 

RESIDUATED MAPS, THE WAY-BELOW RELATION, AND

CONTRACTIONS ON PROBABILISTIC METRIC SPACES

Submitted by

M. Ryan Luke

A Dissertation Approved on

July 3,2017

by the following Dissertation Committee:

Dr. Thomas Riedel, Dissertation Director

Dr. Robert Powers

deceased

Dr. Prasanna Sahoo

Dr. Susan A. Peters 


\title{
DEDICATION
}

To my family, with all my love...

\begin{abstract}
Above all else, I dedicate this work to my loving wife, Leilani Luke. Without her unwaivering support, encouragement, and management of our family, this would not have been possible.
\end{abstract}

I extend my dedication also to my parents, Larry and Maxine, and brother, Stacy who showed unconditional belief in my success and to my mother-in-law, Sharon whose assistance at home has been endless.

Finally, to my sons, Noah, Eli, and James in whom I have more pride than any accomplishments of my own.

God hath no greater blessing than all of you. 


\section{ACKNOWLEDGEMENTS}

I would like to thank my advisor and dissertation director, Dr. Thomas Riedel for tirelessly supporting me in all my endeavors. His wise words have always been sought and appreciated for my research and professional development. I am genuinely grateful for him as a teacher, mentor, colleague, and friend.

I would like to thank the members of my committee, Dr. Robert Powers, Dr. Prasanna Sahoo, and Dr. Susan A. Peters. Not only do I appreciate their service in their role on this committee but also for the additional time that each of them have invested in me and the pursuit of my educational and professional goals through advising and independent study.

Just before defending this dissertation, Dr. Prasanna Sahoo passed away June 18, 2017. This is of great loss to the mathematics community and the University of Louisville. I would like to acknowledge his contribution of over 30 years to the Mathematics Department at University of Louisville and to his students. I have the utmost gratitude for his encouragement and concern for me as a graduate student which were very much a part of my success, and he will be greatly missed.

I would also like to thank the faculty in the Mathematics and Computer Science Departments at Mississippi College. It is because of them that I fell in love with learning and at times wish there were no limits on how many classes you could take. I would like to especially recognize Dr. John McMath; without his encouragement, I would have never continued my graduate study in the first place. I am forever in debt to the time and concern he invested in me, and I am very thankful for his contribution and years of service to me, my fellow students, and 
the math department at Mississippi College.

I am thankful for God and the individual abilities that he gave me. Without His blessing, this also would not be possible.

Finally, I acknowledge that there are many scholars, professors, colleagues, friends, and family who have all played a role in this achievement and its journey. I am thankful for all of those relationships and consider them pillars of my success. 


\title{
ABSTRACT \\ RESIDUATED MAPS, THE WAY-BELOW RELATION, AND CONTRACTIONS ON PROBABILISTIC METRIC SPACES
}

\author{
M. Ryan Luke
}

July 3, 2017

In this dissertation, we will examine residuated mappings on a function lattice and how they behave with respect to the way-below relation. In particular, which residuated $\phi$ has the property that $F$ is way-below $\phi(F)$ for $F$ in appropriate sets. We show the way-below relation describes the separation of two functions and how this corresponds to contraction mappings on probabilistic metric spaces. A new definition for contractions is considered using the way-below relation. 
TABLE OF CONTENTS

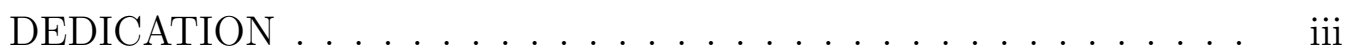
ACKNOWLEDGEMENTS ....................... iv

ABSTRACT ................................. vi

LIST OF FIGURES . . . . . . . . . . . . . . . . . . . viii

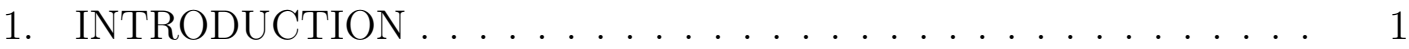

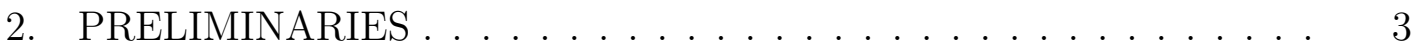

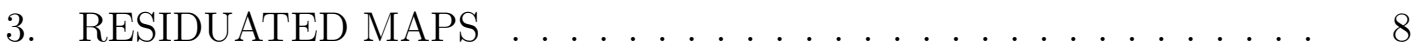

4. THE WAY-BELOW RELATION . . . . . . . . . . . . . . . 13

5. FUNCTION MAPPINGS WITH RESPECT TO WAY-BELOW . . . 27

6. INTRODUCTION TO PROBABILISTIC METRIC SPACES AND CONTRACTION MAPPINGS . . . . . . . . . . . . . . . 44

7. CONTRACTIONS ON PROBABILISTIC METRIC SPACES . . . . 48

8. REMARKS AND CONCLUSION .................. 54

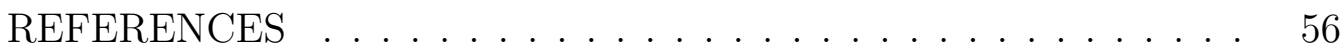
CURRICULUM VITAE ....................... 58 


\section{LIST OF FIGURES}

Figure 2.1. A poset, $P$, of 3 elements . . . . . . . . . . . . 4

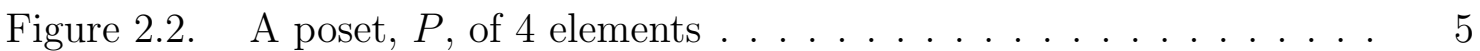

Figure 3.1. The graph of $\delta_{a, b}$, a residuated map . . . . . . . . . . 9

Figure 3.2. The join of two deltas . . . . . . . . . . . . . . . 10

Figure 3.3. The join of two functions in $\operatorname{Res}([0,1]) \ldots \ldots$. . . . . . 11

Figure 4.1. A lattice $L$ of 5 elements . . . . . . . . . . . . . 13

Figure 4.2. An example of deltas where $G$ is way below $F(G \ll F) \quad \ldots \quad 15$

Figure 4.3. An example of deltas that jump to the same value . . . . . . 16

Figure 4.4. An example of deltas that jump at the same point . . . . . . . 17

Figure 4.5. An example of $G$ way below $F(G \ll F) \quad \ldots \ldots \ldots$

Figure 4.6. Residuated maps that are equal at one $x>0 \ldots 20$

Figure 4.7. $F$ and $G$ jump at the same point, $p \ldots \ldots \ldots 21$

Figure 4.8. Alternate example of $F$ and $G$ which jump at the same point . 24 


\section{CHAPTER 1 INTRODUCTION}

The main purpose of this dissertation is to determine how residuated maps behave with respect to the way-below relation, and to use the results obtained to provide a different definition of contraction mapping on probabilistic metric spaces. We begin by examining functions on $\operatorname{Res}(L, M)$ and their behavior under typical lattice operations. Next, we present the way-below relation and how it illustrates the separation of two elements from a lattices as well as some results of cases when those elements are functions themselves; it turns out that the way-below relation is a good way to describe when a function is less than another, and the two are truly separated. In general $f \leq g$ if $f(x) \leq g(x)$ for all $x$ in the domain and $f<g$ if $f \leq g$ and there is an $x_{0}$ such that $f\left(x_{0}\right)<g\left(x_{0}\right)$. However, this does not really separate the graphs of these functions. We will show that the way-below relation in a suitable function space does indeed provide such a separation and use this to the setting of contraction mappings on probabilistic metric spaces.

Most of the basic definitions and preliminary material necessary for reading this dissertation are provided in Chapter 2. Partially ordered sets and lattices have been studied for some time and have been written about at least as early as 1941. A basic introduction to posets and partial order is provided along with the operations join and meet that will be used frequently throughout this text. More specifically, the reader is introduced to lattices, the special case of posets which we use for all our results so far.

Residuated Maps are defined in Chapter 3 and are special nondecreasing 
functions that preserve joins in a lattice and map the bottom element from the lattice of the domain to the bottom element in the lattice of the range. These maps are very closely related to Galois connections from which their study arose but differ in that the composition of two residuated maps is residuated, which is not the case for Galois connections.

Chapter 4 is the beginning of our second part where we present the way-below relation and learn what it means for two elements to be separated. In continuous lattices, elements that have such a relationship (other than those way-below themselves such as 0) are separated enough that another element can be found between them. While we present this relation in general, we are more concerned about its application to functions and what is required for a function to be way-below another function.

We also answer the question in Chapter 5 about how mappings in $\operatorname{Res}([p, q],[r, s])$ affect the way-below relation. The results presented classify for which $F \in \operatorname{Res}([p, q],[r, s])$ is $\phi(F)$ way-below $F$ or $F$ way-below $\phi(F)$ when $\phi: \operatorname{Res}([p, q],[r, s]) \rightarrow \operatorname{Res}([p, q],[r, s])$. We also discuss whether a way-below realtionship is preserved when applying $\phi$ to two functions that have such a relationship.

In the last part of this dissertation beginning with Chapter 6, we draw a connection to probabilistic metric spaces (PM spaces) and contraction mappings. The definition of PM spaces, distance distributions functions, triangle functions, and contraction mappings are all provided and discussed in Chapter 6 .

We introduce existing results on contraction mappings in Chapter 7 and provide a new definition using the way-below relation. We then discuss our findings and compare them to those of T.L. Hicks, V.M. Sehgal, and A.T. Bharucha-Reid.

Finally in Chapter 8, we make some closing remarks about our observations and provide potential avenues for further research. 


\section{CHAPTER 2 \\ PRELIMINARIES}

In order to keep this dissertation reasonably self-contained, we start by providing the definitions of some necessary terms as well as some well-known results. Much of this can be found in references [1] and [3] or other introductory books on order. First and foremost, we define the basic structures of order theory: partially ordered sets and lattices.

DEFINITION 2.1 (partial order; partially ordered set). Let $P$ be a set. A partial order on $P$ is a binary relation $\leq$ on $P$ that is reflexive, antisymmetric, and transitive. That is, for all $x, y, z$ in $P$,

(i) $x \leq x$,

(ii) $x \leq y$ and $y \leq x$ imply $x=y$,

(iii) $x \leq y$ and $y \leq z$ imply $x \leq z$.

$A$ set $P$ equipped with a partial order is called a partially ordered set, or poset for short.

A partially ordered set is a set along with a binary relation that describes how some elements compare to other elements in the set, but every element in a poset is not necessarily related to every other element in the poset, thus a partial order. In fact, two elements $a$ and $b$ that are not related are called non-comparable, and we denote this $a \| b$. A poset in which every two elements are related is called a totally ordered set or chain. 
For example, the set of Real Numbers $(\mathbb{R})$ with the standard order, is a totally ordered set and so are any of its subsets. For any two real numbers, we have the law of trichotomy: $a=b, a<b$ or $b<a$.

We often (and in this dissertation) use the notion of posets to examine sets of elements that have a more complex relationship or are not just numbers, such as a set of functions. Like with numbers, it makes sense that we may also define two operations for posets.

DEFINITION 2.2 (Join). The join of a subset, $S$, of a poset, $P$, is the supremum (or least upper bound) and denoted, $\vee S$, if it exists.

We take note that the join of $S$ does not have to be in $S$ but it can be. For example, if we take $S=\{x \in \mathbb{R} \mid x<1\}$, then $\bigvee S=1$ and $1 \in \mathbb{R}$ but $1 \notin S$. Alternately, if $S=\{1,2\} \subset \mathbb{R}$, then $\bigvee S=2$. Furthermore, the join does not necessarily exist. For example, consider the poset in Figure 2.1 where if $S=P$, then $\bigvee S$ does not exist:

Figure 2.1: A poset, $P$, of 3 elements

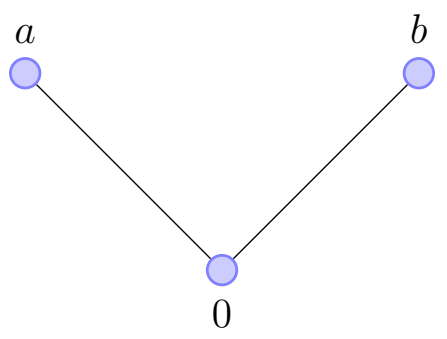

We also define the operation for greatest lower bound in a poset.

DEFINITION 2.3 (Meet). The meet of a subset, $S$, of a poset, $P$, is the infimum (or greatest lower bound) and denoted, $\wedge$ S, if it exists.

Similarly to the join, the meet of $S$ need not be in $S$. Again consider $P=\mathbb{R}$ and let $S=\{x \in \mathbb{R} \mid x>0\}$, then $\wedge S=0$ and $0 \in \mathbb{R}$ but $0 \notin S$. The meet also does not 
necessarily exist and we can consider the poset in Figure 2.2 where if $S=P$, then $\wedge S$ does not exist:

Figure 2.2: A poset, $P$, of 4 elements

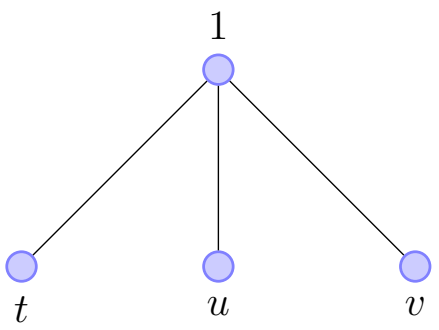

If $S=\left\{a_{1}, a_{2}, \ldots, a_{n}\right\}$, we often write $\vee S=a_{1} \vee a_{2} \vee \ldots \vee a_{n}$ and $\wedge S=a_{1} \wedge$ $a_{2} \wedge \ldots \wedge a_{n}$. Looking again at the previous diagrams, in Figure 2.1 for $S=\{a, b\}$ we have $\wedge S=a \wedge b=0$, and in Figure 2.2 for $S=\{t, u, v\}$ we have $\vee S=t \vee u \vee v=1$. Posets in which both the join and the meet exist for every pair of elements are a special class.

DEFINITION 2.4 (Lattice). A lattice is a poset in which any two elements have a least upper bound (join) and greatest lower bound (meet).

Davey and Priestly [3] remind us that prime numbers are product-irreducible natural numbers (other than 1) and that we have an analogous idea in lattices.

DEFINITION 2.5 (join-irreducible). Let $L$ be a lattice. An element $x \in L$ is joinirreducible if $x \neq 0_{L}$ and $x=a \vee b$ implies $x=a$ or $x=b$ for all $a, b \in L$.

DEFINITION 2.6 (meet-irreducible). Let $L$ be a lattice. An element $x \in L$ is meetirreducible if $x \neq 1_{L}$ and $x=a \wedge b$ implies $x=a$ or $x=b$ for all $a, b \in L$.

We also consider special classes of lattices. A lattice in which every subset has a meet and a join is called a complete lattice. It should be noted that to be a complete lattice the empty set, $\varnothing$, must also have a join and a meet. To explain how this works, we define top and bottom elements of a poset. 
DEFINITION 2.7 (top element). Let $P$ be a poset. We say $P$ has a top element or one if there exists $1 \in P$ (called top) with the property that $x \leq 1$ for all $x \in P$.

DEFINITION 2.8 (bottom element). Let $P$ be a poset. We say $P$ has a bottom element or zero if there exists $0 \in P$ (called bottom) with the property that $0 \leq x$ for all $x \in P$.

Top and bottom elements (i.e. 1 and 0 ) are required for finite posets to be lattices while infinite lattices require them for completeness.

For example, in a finite poset (i.e. poset with a finite number of elements) such as the one shown in Figure 2.1, the join of $a$ and $b$ needs to exist for $P$ to be considered a lattice. If $a \vee b$ did exist in this diagram, it would be the top element and $P$ would be a lattice. Likewise in Figure 2.2, the pairwise meet of $t, u$, and $v$ must exist and would be the bottom element. The lattices created in these two examples are known as $M_{2}$ and $M_{3}$. In fact $M_{n}$ is a family of lattices that consist of a 1 and 0 with $n$ non-comparable elements each of which are connected only to the 1 and 0 .

The 1 and 0 are required for an infinite lattice to be a complete lattice. This is because the definition requires every subset (including the empty set and entire lattice) to have a join and meet. The top element is required for completeness because the entire lattice must have a join, and the bottom element is required for completeness because the entire lattice must have a meet.

It is interesting to also note that the join of the empty set is the bottom element, and the meet of the the empty set is the top element. In particular, this means that the join is not always greater than the meet.

There are other special classes of lattices, one of which we consider in much of the research contained in this dissertation. We now define distributive lattices.

DEFINITION 2.9 (distributive lattice). A lattice, $L$ is said to be distributive if 
it satisfies the distributive law, that is, for all $a, b, c \in L$, we have $a \wedge(b \vee c)=$ $(a \wedge b) \vee(a \wedge c)$

For the remainder of this dissertation, we will assume the lattices that we refer to are complete, distributive lattices, but we will often need to place further restrictions on them. 


\section{CHAPTER 3 RESIDUATED MAPS}

Much of this dissertation is concerned with particular function lattices. We examine $\operatorname{Res}(L, M)$, the functions it contains, and the behavior of these functions under operations on the lattice. $\operatorname{Res}(L, M)$ is the set of residuated maps from lattice $L$ to lattice $M$, and we provide its formal definition below. While we restrict our discussion to lattices, residuated maps can be formally defined on partially ordered sets. The following definition can be found in [1]. In this context, $f$ is nondecreasing if $x<y$ in the domain implies $f(x) \leq f(y)$ in the range.

DEFINITION 3.1 (residuated map). If $L, M$ are partially ordered sets, a map $f: L \rightarrow M$ is residuated if and only if $f$ is isotone (nondecreasing) and there exists a unique, isotone map $f^{+}: M \rightarrow L$ such that $f^{+} \circ f \geq i d_{L}$ and $f \circ f^{+} \leq i d_{M}$. The unique map $f^{+}$, called the residual of $f$, is defined such that $\forall y \in M, f^{+}(y)=\bigvee\{x \epsilon$ $L \mid f(x) \leq y\}$.

REMARK 3.2. A function, $f$, is in Res $(L, M)$ if it has the following properties:

- $f(0)=0 \quad$ (i.e. maps the bottom element of $L$ to the bottom element of $M$ )

- $f$ is non-decreasing

- $f$ preserves arbitrary joins (i.e. $f(a \vee b)=f(a) \vee f(b)$ )

If $L$ and $M$ are complete, distributive lattices and $S$ is any subset of $L$, we get $f(\bigvee S)=\vee f(S)$. 
If $L, M$ are real intervals, then $f$ is a nondecreasing function which maps the left end point of $L$ to the left end point of $M$ and is left continuous.

Figure 3.1: The graph of $\delta_{a, b}$, a residuated map

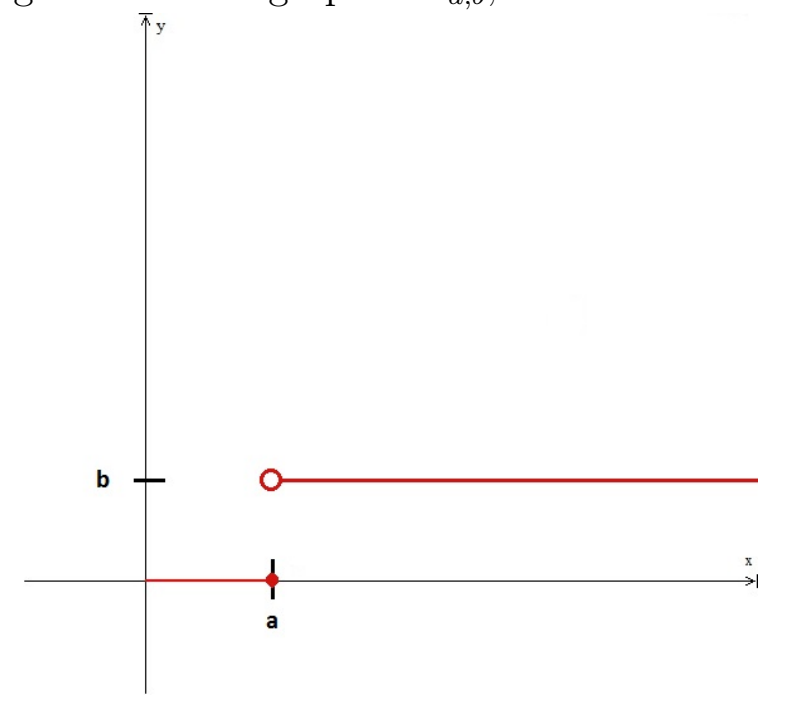

The simplest of such functions are ones that take on only two values, zero and one other. Figure 3.1 shows the graph of one, and we define them in this way:

$$
\delta_{a, b}(x)= \begin{cases}0 & \text { if } x \leq a \\ b & \text { otherwise }\end{cases}
$$

Very often, we begin working on a problem or hypothesis by using deltas. Since deltas are residuated and very easy to evaluate, we can sometimes find out very quickly how something behaves or if it doesn't work at all. Beyond the ease of using them, deltas can also be used to construct any other residuated map.

We introduced the notion of joins in Chapter 2 and we must become aware of how they work when the elements are functions in $\operatorname{Res}(L, M)$. For joins of functions, the operation is pointwise. This means, that the join is evaluated point by point in the domain.

We first look at the join of two delta functions. 
EXAMPLE 3.3. Consider Res([0,1]):

$$
\delta_{\frac{1}{2}, \frac{1}{2}} \quad \vee \quad \delta_{\frac{3}{4}, \frac{3}{4}}= \begin{cases}0 & \text { if } x \leq \frac{1}{2} \\ \frac{1}{2} & \text { if } \frac{1}{2}<x \leq \frac{3}{4} \\ \frac{3}{4} & \text { if } x>\frac{3}{4}\end{cases}
$$

Recall that the join of a finite set of real numbers takes the largest value at each point. Thus, for any $x \leq \frac{1}{2}$, both of the above functions are 0 and $0 \vee 0=0$. For values of $x$ that are between $\frac{1}{2}$ and $\frac{3}{4}$, the function on the left is $\frac{1}{2}$ and the function on the right is 0 and so $\frac{1}{2} \vee 0=\frac{1}{2}$. Finally for $x>\frac{3}{4}$, both functions achieve their highest values and $\frac{1}{2} \vee \frac{3}{4}=\frac{3}{4}$. The result shown in Figure 3.2 is actually a step function as given above.

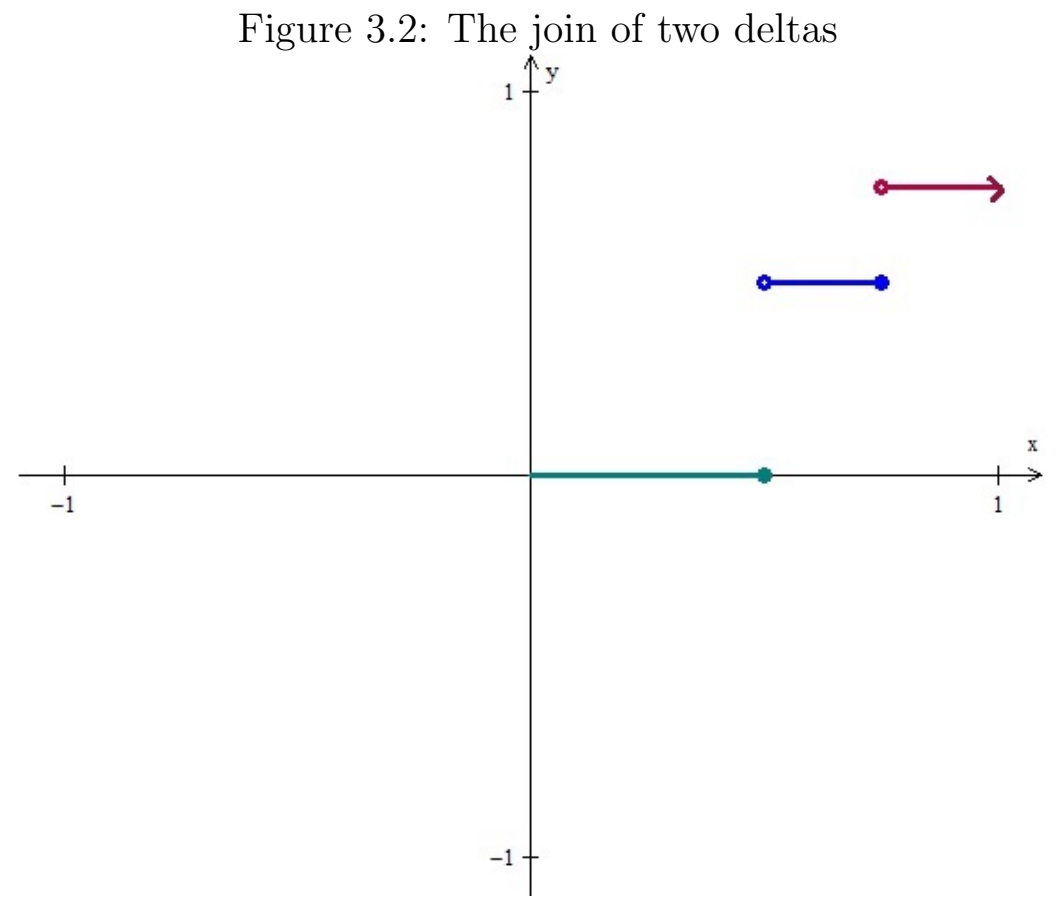

Using this example, we see that joins behave point-wise on deltas. This is the same for arbitrary functions since any function can be written as the join of deltas. When $\operatorname{Res}(L, M)$ is distributive, deltas are the join irreducibles, and a function, $f$ can be written as the join of join irreducibles. 
Figure 3.3: The join of two functions in $\operatorname{Res}([0,1])$
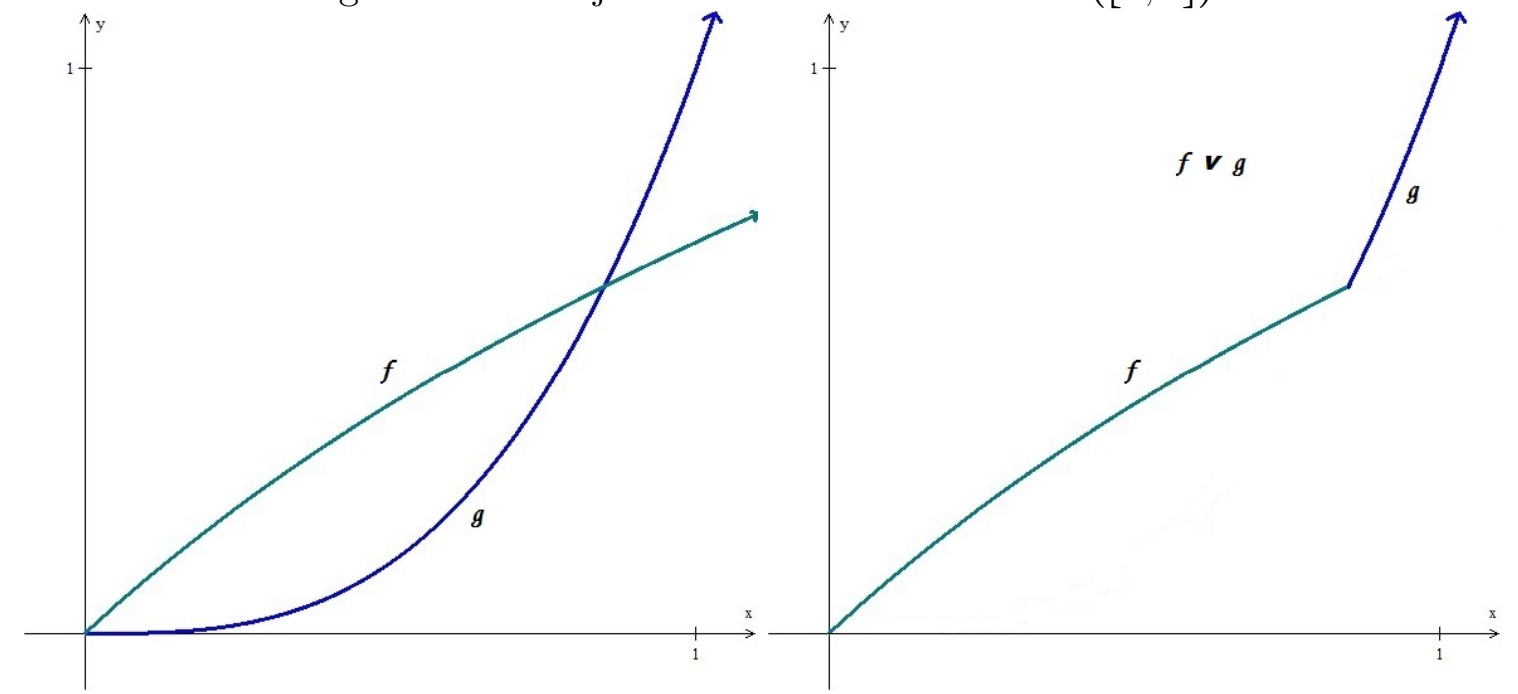

One can see in Figure 3 that the graph on the right is the join of the 2 graphs on the left and is formed by taking the highest value of the two functions at each point.

It is important to note that $\delta_{0,0}$ is the bottom element and $\delta_{0,1}$ is the top element of $\operatorname{Res}([0,1])$. Because it is equivalent to the zero function, there is no function smaller than $\delta_{0,0}$ in $\operatorname{Res}([0,1])$. The largest value a function can take in $\operatorname{Res}([0,1])$ is 1 , but every function in $\operatorname{Res}([0,1])$ has to be 0 at 0 . Thus, the largest function that satisfies these requirements is $\delta_{0,1}$, making it the top element.

Another important characteristic of a residuated map, $F$, that we use and refer to often in this dissertation is the least upper bound of points where $F$ takes the value zero. More formally, this point is denoted $b_{F}$ and defined this way:

$$
b_{F}=\bigvee\{x \mid F(x)=0\}
$$

This is the notation that will be used in this dissertation, but it should be noted that it can be defined equivalently using the residual of $F$ from Definition 3.1:

$$
b_{F}=\bigvee\{x \mid F(x)=0\}=F^{+}(0)
$$


Now that we have an idea of the nature of residuated maps, our intention is to actually look at mappings from a set of residuated maps to another set of residuated maps. Consider,

$$
\phi: \operatorname{Res}([0,1]) \rightarrow \operatorname{Res}([0,1])
$$

What properties must $\phi$ have so that

$$
\phi \in \operatorname{Res}(\operatorname{Res}([0,1])) \quad ?
$$

Like all residuated mappings, we require $\phi$ to map the bottom element of the domain to the bottom element in the range and it must preserve arbitrary joins. Explicitly, let $\mathcal{A}$ be an index set and $F_{\alpha} \in \operatorname{Res}([0,1])$ for all $\alpha \in \mathcal{A}$, then

$$
\begin{aligned}
\phi\left(\delta_{0,0}\right) & =\delta_{0,0} \\
\phi\left(\bigvee_{\alpha \in \mathcal{A}} F_{\alpha}\right) & =\bigvee_{\alpha \in \mathcal{A}} \phi\left(F_{\alpha}\right) \\
\Longrightarrow \phi & \in \operatorname{Res}(\operatorname{Res}([0,1]))
\end{aligned}
$$




\section{CHAPTER 4 THE WAY-BELOW RELATION}

In this chapter, we discuss the way-below relation. It can provide us insight on how separated two elements in a lattice are. For function lattices, e.g. Res $(L)$ or $\operatorname{Res}(\operatorname{Res}(L))$, if one function is way-below another function, we can find at least one function that is strictly between them; this notion of strict is what we discuss here.

To formally define the way-below relation, we must first be familiar with directed sets.

Definition 4.1 (Directed Set). Let $D$ be a subset of a poset. $D$ is directed if every finite subset of $D$ has an upper bound in $D$.

For example, every chain is a directed set. For another example, we consider the lattice in Figure 4.1.

Figure 4.1: A lattice $L$ of 5 elements

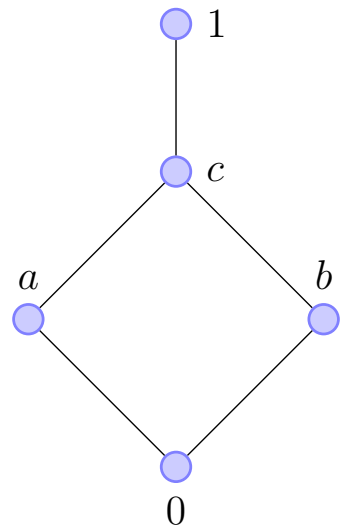


In Figure 4.1, the set $\{a, b, 1\}$ is directed since the upper bound 1 is in the set. Alternately, $\{a, b\}$ is not a directed set since the upper bound of $a$ and $b$ is $c$, but $c$ is not in the set $\{a, b\}$.

With an understanding of directed sets, the formal definition of the waybelow relation is now provided.

DEFINITION 4.2 (The Way-Below Relation). Let $x$ and $y$ be elements of a lattice, L. Then $x$ is way-below $y$, denoted $x \ll y$, if for every directed subset $D$ of $L$ such that $y \leq \vee D$, there exists an element $d \in D$ such that $x \leq d$.

Like with residuated maps, we give the bottom element, or 0 , of the lattice some special attention.

REMARK 4.3. In any lattice, L, the bottom element 0 is way-below itself. (i.e. $0 \ll 0)$.

Proof. Let $D$ be a directed set in $L$ such that $\vee D=0$. Since 0 is the bottom element of $L$, then for all $d \in D$, we have $0 \leq d \leq 0=\bigvee D$. Therefore, $0 \in D$ and $0 \ll 0$.

REMARK 4.4. We also note that elements that are way-below themselves, such as the bottom element, are commonly referred to as being compact.

The Way-Below relation intuitively implies a less-than / greater-than relationship. If $x$ is way-below $y$, then most certainly $x \leq y$.

LEMMA 4.5. Whenever $x \ll y$ we have $x \leq y$.

Proof. Suppose not. That is, suppose $x \nless y$. Consider $D=\{y\}$, a directed set. Then, $\vee D=y \geq y$. However, $y ¥ x$, hence, $x \nless k y$, a contradiction.

Later, we will conclude that for residuated maps, this condition is actually stronger. When the value of one function is no longer 0 , a function that is waybelow must be strictly less than the other function. We have to look at where the 
functions are not equal to 0 since all residuated maps are required to be 0 at least at 0 .

Since any two functions in $\operatorname{Res}(L, M)$ must map $0_{L}$ to $0_{M}$, we have that no two functions, $f, g$ can satisfy $f(x)<g(x)$ for all $x$ in $L$. It turns out that it is not sufficient to assume $f(x)<g(x)$ for all $x$ in $L \backslash\left\{0_{L}\right\}$ to attain $f \ll g$. Much of this dissertation is devoted to studying the way-below relation on $\operatorname{Res}(L, M)$.

With the formal definition and conceptual ideas having been discussed, it is natural to now provide a graphical explanation of the way-below relation on $\operatorname{Res}(L, M)$. As mentioned before, the notion of way below can be seen as a separation of the two elements. So, in our case, on lattices of residuated maps, we examine functions that are way below other functions, and we will show that there is room between the two functions to squeeze in a third function.

As with most ideas that we have involving residuated maps, it makes sense that we first take a look at deltas. Figure 4.2 is an example of two deltas in which $G$ is way below $F$.

Figure 4.2: An example of deltas where $G$ is way below $F(G \ll F)$

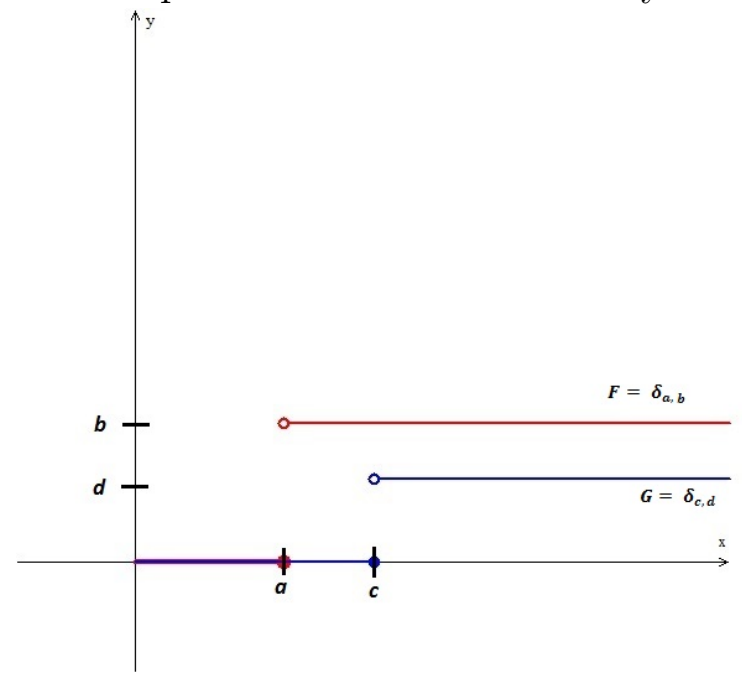

There are few things to notice here in Figure 4.2 about the relationship between $F=\delta_{a, b}$ and $G=\delta_{c, d}$ where $a<c$ and $b>d$. First, as required by Lemma 
4.5, $G \leq F$. Another thing is that $G$ has the value of 0 longer than $F$, or as discussed in Equation 3.1, we say $b_{G}>b_{F}$. If $G$ left zero before $F$, then $G \not F$, and we will discuss what happens if $G$ and $F$ leave zero at the same point. Finally, we also take note that $F$ jumps to a higher value than that of $G$ (i.e. $F(x)>G(x)$ for $\{x \mid F(x) \neq 0\})$.

One must then ask the questions regarding whether a way-below relationship is maintained, "Can both $F$ and $G$ jump at the same point?" and "Can both $F$ and $G$ jump to the same value?" These are valid points that should be investigated since neither of these situations would violate Lemma 4.5.

Figure 4.3: An example of deltas that jump to the same value

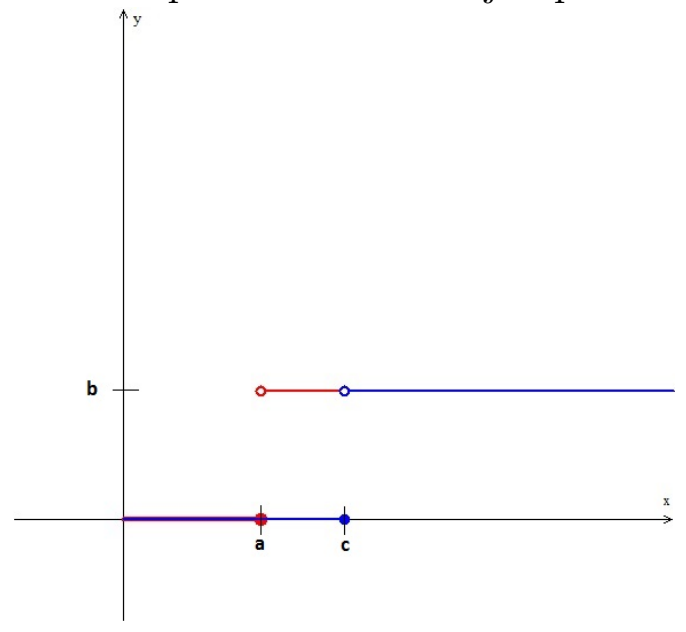

The functions $\delta_{a, b}$ and $\delta_{c, b}$ jump to the same height, $b$, as shown in Figure 4.3, and $\delta_{c, b} \leq \delta_{a, b}$ However, this is not a sufficient condition to be way-below. In fact, the functions in Figure 4.3 do not have a way-below relationship. Consider the directed set $D=\left\{\delta_{a, b-\frac{1}{n}}\right\}$. The join of $D$ is most certainly $\delta_{a, b}$ but not a single element in $D$ is greater than or equal to $\delta_{c, b}$ and thus does not meet the definition of way-below.

When deltas jump at the same point (as in Figure 4.4), they have a strictly less-than relationship everywhere after that. This poses a problem for being waybelow as well. While $\delta_{u, v} \leq \delta_{u, t}$ and $\delta_{u, v}(x)<\delta_{u, t}(x)$ for $x>u$, this just isn't enough. 
Consider the directed set $D=\left\{\delta_{u+\frac{1}{n}, t}\right\}$. The join of $D$ is $\delta_{u, t}$ but no element in $D$ is greater than or equal to $\delta_{u, v}$. Again, this does not satisfy the definition of way-below.

REMARK 4.6. Note that while the pictures are for $c>0$ in Figure 4.3 and $u>0$ in Figure 4.4, the same arguments work if a or u equal zero. This means that any residuated map that is zero only at zero and nonzero everywhere else cannot be way-below another function. For example, $\delta_{0, z}$ for $z>0$ is not way-below anything.

Figure 4.4: An example of deltas that jump at the same point

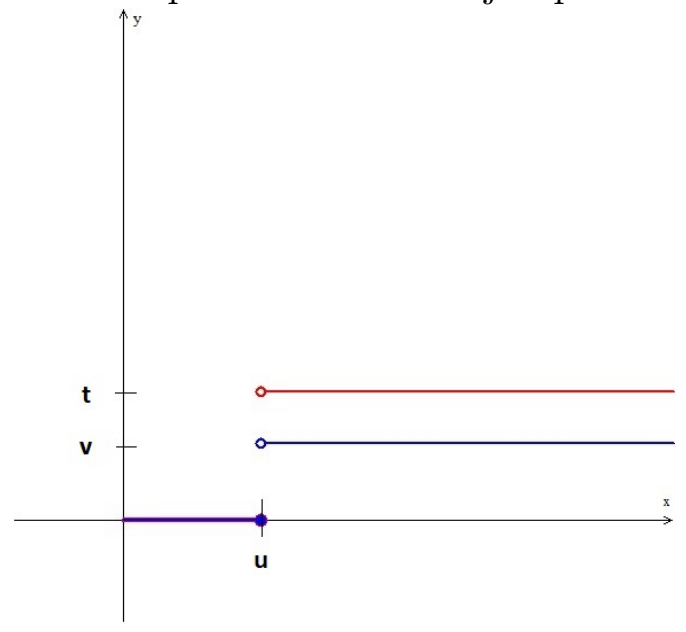

So, at the very least, we know that for a delta to be way-below another delta, the delta that is way-below cannot jump as high and must jump later. Graphically speaking, the delta that is way above should be shifted left and up. These conditions are not only necessary but sufficient.

THEOREM 4.7. Let $\delta_{a, b}$ and $\delta_{c, d}$ be in Res $(L)$. Then $\delta_{a, b}$ is way below $\delta_{c, d}$ if and only if $a>c$ and $b<d$.

Proof. If $a<c$ or $b>d$, then $\delta_{a, b} \nless \delta_{c, d}$ which is a contradiction to Lemma 4.5 .

If $a=c$ and $b \leq d$, then $\delta_{a, b}$ and $\delta_{c, d}$ jump at the same point and we consider the directed set $D=\left\{\delta_{c+\frac{1}{n}, d}\right\}$. We have $\bigvee D=\delta_{c, d}$, however, $\delta_{a, b} \nless d$ for any $d \in D$. Thus, 
$\delta_{a, b} \nless k \delta_{c, d}$.

If $b=d$ and $a>c$, then $\delta_{a, b}$ and $\delta_{c, d}$ jump to the same height and we consider the directed set $D=\left\{\delta_{c, d-\frac{1}{n}}\right\}$. We have $\bigvee D=\delta_{c, d}$, however, $\delta_{a, b} \nless d$ for any $d \in D$. Thus, $\delta_{a, b} \nless k \delta_{c, d}$.

Therefore, by contrapositive argument,

$$
\delta_{a, b} \ll \delta_{c, d} \Longrightarrow a>c \text { and } b<d
$$

For the other direction, assume that $a>c$ and $b<d$. This gives us $\delta_{a, b} \leq \delta_{c, d}$. Now, suppose $D$ is directed and $\bigvee D=\bigvee_{F \in D} F \geq \delta_{c, d}$. This implies that there is an $x_{0}$ in $(c, a)$ and an $F_{0}$ in $D$ such that

$$
b<F_{0}\left(x_{0}\right)
$$

Since $a>x_{0}$ and $F_{0}$ is nondecreasing, we get

$$
F_{0}(a) \geq F_{0}\left(x_{0}\right)>b
$$

So, for this $F_{0}$ in $D$, we have

$$
F_{0}(x) \geq b \text { for all } x \geq a
$$

which implies

$$
F_{0} \geq \delta_{a, b}
$$

That is, for any such $D$, there exists an $F \in D$ such that $\delta_{a, b} \leq F$ and therefore

$$
\delta_{a, b} \ll \delta_{c, d}
$$

In order for a delta to be way below another delta, it must remain zero longer and must jump to a height strictly less than the other delta. It is reasonable to assume that this extends to general residuated maps. Since general residuated maps may take on many values, it is natural that many other questions arise. 
The following picture, Figure 4.5, is an example of two residuated maps in which the function on the bottom $(G)$ is way-below the function on the top $(F)$. The important thing to notice is that they are only equal at the beginning where they are both equal to zero. After the function $F$ leaves 0 , the graphs maintain a strictly less than relationship $(G<F)$ yielding definite separation between the two in which another function could be drawn. It is also important to notice, albeit a direct consequence to what was already stated, that the function that is way-below, $G$, stays 0 (value is zero) longer, just as deltas did.

Figure 4.5: An example of $G$ way below $F(G \ll F)$

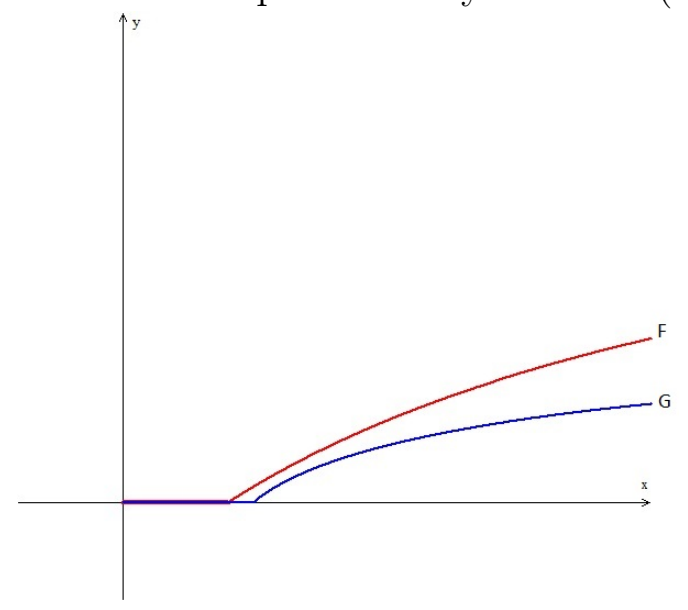

These observations beg the questions, "Can the functions ever intersect at a value other than 0 and still have a way-below relationship?" and "Can they leave zero at the same time and have a way-below relationship?

Since deltas take on a finite number of values, namely two - zero and its second index, we ask if this affects the strictly less than restriction after leaving zero or if this must be true for all residuated maps in general. We consider two residuated maps, $F$ and $G$, such that $F(p)=G(p)$ and $F(x)<G(x)$ for all $x>0$ and $x \neq p$. These functions are illustrated by Figure 4.6. As it turns out, this is still problematic. 
Figure 4.6: Residuated maps that are equal at one $x>0$

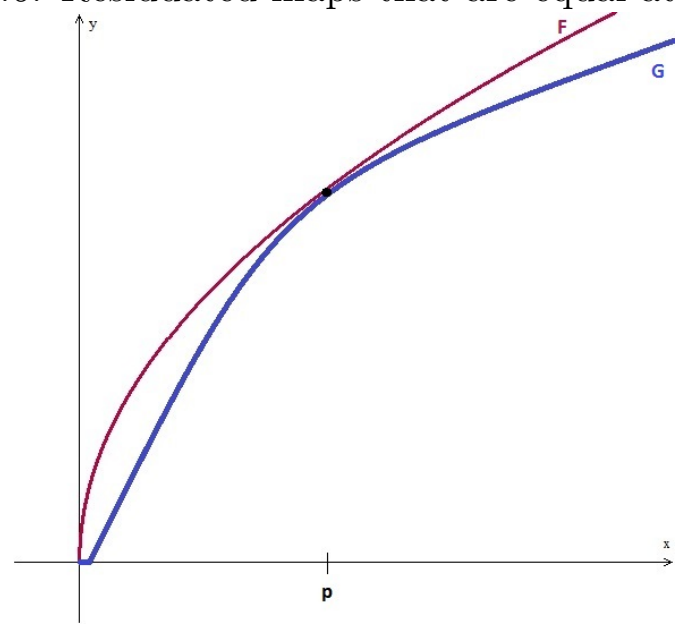

Consider the functions,

$$
H_{n}(x)= \begin{cases}F(x) & 0 \leq x \leq p-\frac{1}{n}, \\ F\left(p-\frac{1}{n}\right) & p-\frac{1}{n}<x \leq p+\frac{1}{n}, \\ F(x) & p+\frac{1}{n} \leq x\end{cases}
$$

Then,

$$
D=\left\{H_{n} \mid n \in \mathbb{N}\right\}
$$

is directed and $\bigvee D=F$. However, for each $n$,

$$
H_{n} \| G
$$

and therefore,

$$
G \nless k F \text {. }
$$

So, we conclude the functions must maintain a strictly less than relationship and this argument is analogous to the one discussed about Figure 4.3 where two deltas jump to the same height.

The other question that needs to be answered is about jump discontinuities. Deltas jump from zero to only one other value, but general residuated maps can 
jump multiple times and from values other than zero. Does this affect the restrictions about jumping at the same point? In Figure 4.7, we see an example of residuated maps, $F$ and $G$, that jump at the same point and yet $G<F$ for all $x>0$ not just after its jump point.

Figure 4.7: $F$ and $G$ jump at the same point, $p$

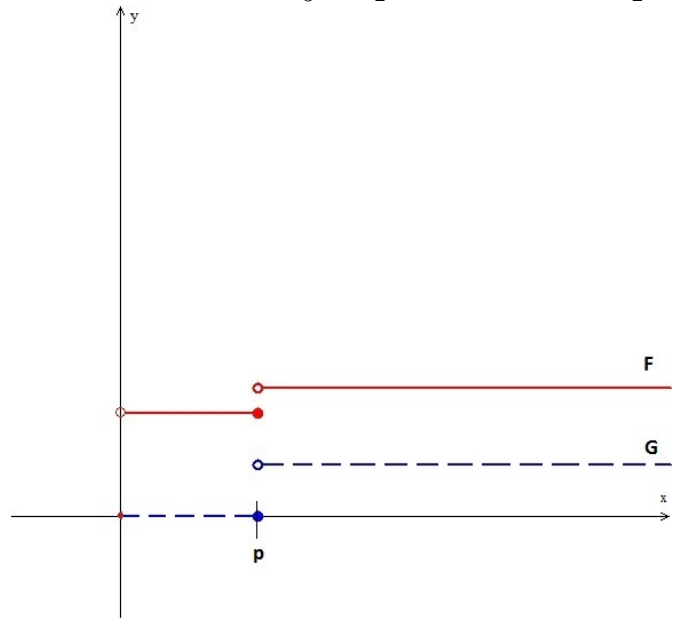

We answer these questions more formally in the proofs that follow. To begin, we consider what is required for a delta to be way-below an arbitrary residuated function. We have shown that in order to have a way-below relationship, a delta that is way-below cannot leave zero at the same time as the other function and that, when it leaves zero, it must take a value that is strictly less than the other function. This gives us the following theorem.

THEOREM 4.8. Let $\delta_{a, b}$ and $F \in \operatorname{Res}([p, q],[r, s])$ then $\delta_{a, b} \ll F$ if and only if $a>b_{F}=\bigvee\{x \mid F(x)=0\}$ and $b<F(a)$

Proof. For the only if, suppose $\delta_{a, b} \ll F$. By Lemma 4.5, we have

$$
\delta_{a, b} \leq F
$$

This implies that

$$
a \geq b_{F} .
$$


We also want to show that $b<F(a)$. Then, again by way of contradiction, assume that $b \geq F(a)$.

$$
d_{n}(x)= \begin{cases}0 & \text { if } x=0 \\ b-\frac{1}{n} & \text { if } 0<x \leq a+\frac{1}{n} \\ F(x) & \text { if } x>a+\frac{1}{n}\end{cases}
$$

Again, $D=\left\{d_{n} \mid n \in \mathbb{N}\right\}$ is a directed set since it forms a chain and $\bigvee D \geq F$. However,

$$
d_{n} \| \delta_{a, b} \text { for all } n \text {. }
$$

So, $\delta_{a, b} \nless d_{n}$ for all $n \in \mathbb{N}$. This contradicts $\delta_{a, b}<<F$. Therefore,

$$
\delta_{a, b} \ll F \text { implies } a>\bigvee\{x \mid F(x)=0\}=b_{F} \text { and } b<F(a)
$$

Now, for the reverse direction, suppose $a>\bigvee\{x \mid F(x)=0\}$ and $b<F(a)$

Let $D$ be an arbitrary directed set where $\vee D=F$. We want to show that there is a $d \in D$ such that $d \geq \delta_{a, b}$. Suppose that $d \nsucceq \delta_{a, b}$ for all $d \in D$.

Since

$$
d \geq \delta_{a, b} \Longleftrightarrow \lim _{x \rightarrow a^{+}} d(x) \geq b
$$

we get

$$
d \nsucceq \delta_{a, b} \Longleftrightarrow \lim _{x \rightarrow a^{+}} d(x)<b .
$$

This implies, since $d$ is non-decreasing that

$$
d(a) \leq \lim _{x \rightarrow a^{+}} d(x)<b, \text { for all } d \in D .
$$

which means $(\bigvee D)(a) \leq b$. Since $\bigvee D=F$, we get $(\bigvee D)(a)=F(a)$ but by assumption we have $F(a)>b$, thus yielding a contradiction and the theorem holds. 
Theorem 4.8 formalizes the idea that for a function to be way below, it must stay at zero longer and after leaving zero must remain strictly less than the other function.

It should be pointed out that this also means both functions cannot leave zero immediately, i.e. $G \ll F$ does not allow $b_{G}=0$. While all residuated maps are zero at zero, a function, $G$, that is way-below another function has to be zero more than just at that single point, so $b_{G}>0$.

From the details provided so far such as requiring the lower function to remain zero longer, it may seem reasonable that a way-below relationship prevents two functions from sharing a jump discontinuity (Compare Figure 4.7 and Figure 4.8). This is not the case. Recall Figure 4.7 and notice that $G=0$ longer than $F\left(b_{G}>b_{F}\right)$ and that $G<F$ for all $x>0$. From this and the proofs so far, we can see that it is not necessarily the "jump" but what happens at that point of discontinuity that poses a problem. We now examine the graphs in Figure 4.8. In contrast to Figure 4.7, these two functions do not have a way-below relationship even though $\left(b_{G}>b_{F}\right)$ and that $G<F$ for all $x>0$. Consider the directed set

$$
D=\left\{d_{n}(x)=\left\{\begin{array}{ll}
F\left(x-\frac{1}{n}\right) & \text { for } x>\frac{1}{n} \\
0 & \text { otherwise }
\end{array} \mid \text { for all } n \in \mathbb{N}\right\}\right.
$$

$G$ is not less than or equal to any $d$ in $D$, specifically because of what happens at point $p$.

It is evident now that the jump discontinuity is not the problem per se, but it does become a point of interest; the overlapping situation shown in Figure 4.8 cannot happen if $G$ is to be way-below $F$. More formally, in order for a way-below relationship to exists, we must have

$$
F(p)=\lim _{x \rightarrow p^{-}} F(x)>\lim _{x \rightarrow p^{+}} G(x) .
$$

LEMMA 4.9. Suppose $F, G \in \operatorname{Res}([p, q],[r, s])$, then 
Figure 4.8: Alternate example of $F$ and $G$ which jump at the same point

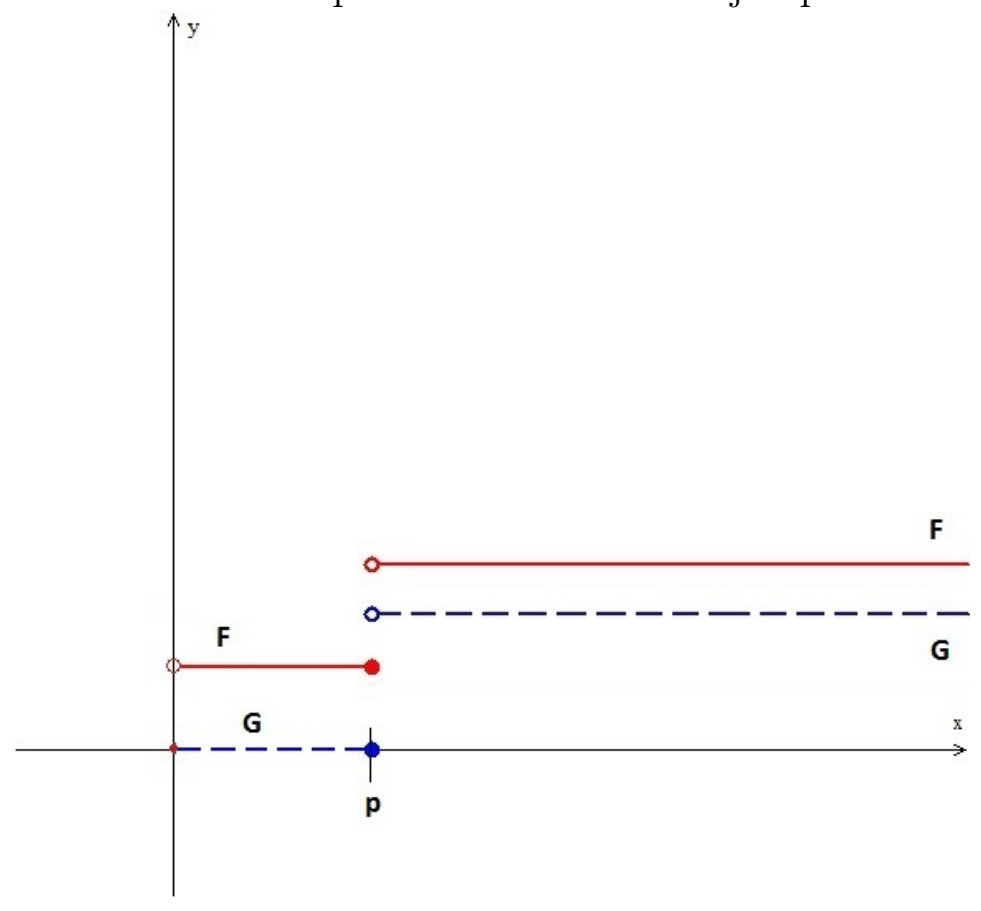

a) $b_{G}>b_{F}$ and $G(q)<F(q)$ and

b) $G^{r}(x)=\lim _{t \rightarrow x+} G(t)<F(x)$ for all $x \in\left[b_{G}, q\right)$

imply that $m=\inf \left\{F(x)-G^{r}(x) \mid x \in\left[b_{G}, q\right]\right\}>0$.

Proof. We know the infimum exists since the set is non empty and bounded below by 0 . Thus there is a sequence $\left\{x_{n}\right\}_{n \in \mathbb{N}}$ in $\left(b_{G}, q\right]$ such that $\left(F-G^{r}\right)\left(x_{n}\right)$ converges toward this infimum $m$. Since the sequence is bounded, there is a convergent subsequence $\left\{x_{n_{j}}\right\}_{j \in \mathbb{N}}$ of the $\left\{x_{n}\right\}_{n \in \mathbb{N}}$ converging to some $t \in\left[b_{G}, q\right]$ and this subsequence can be chosen to be decreasing or increasing (if infinitely many elements of the original sequence are less than the limit, we have an increasing sequence, otherwise a decreasing one). If the subsequence is increasing, then since our functions are left continuous we get $0<F(t)-G^{r}(t) \leq F(t)-G(t)=\lim _{j \rightarrow \infty}(F)\left(x_{n_{j}}\right)-\lim _{j \rightarrow \infty} G^{r}\left(x_{n_{j}}\right)=m$. Similarly if the sequence is decreasing we get $0<F(t)-G^{r}(t) \leq F^{r}(t)-G^{r}(t)=$ $\lim _{j \rightarrow \infty} F\left(x_{n_{j}}\right)-\lim _{j \rightarrow \infty} G^{r}\left(x_{n_{j}}\right)=m$ hence the infimum has to be positive and the graphs of $F$ and $G$ are vertically separated. 
This allows us to now clearly formulate how much two functions are separated when they are way-below one another.

THEOREM 4.10. Suppose $F, G \in \operatorname{Res}([p, q],[r, s])$, and let

a) $b_{G}>b_{F}$ and $G(q)<F(q)$

b) $G^{r}(x)=\lim _{t \rightarrow x+} G(t)<F(x)$ for all $x \in\left[b_{G}, q\right)$

Then $G \ll F$ if and only if a) and b) hold.

Proof. By several previous examples we have seen that $G \ll F$ implies conditions a) and b). For the converse, we will construct a collection $\left\{\delta_{x_{n}, y_{n}} \mid n=1, \ldots, N\right\}$ such that $\delta_{x_{n}, y_{n}} \ll F$ for $n=1, \ldots, N$ and $G \leq \bigvee_{n=1}^{N} \delta_{x_{n}, y_{n}}$. Let $x_{1}=b_{G}$ and $y_{1}=G^{r}\left(x_{1}\right)+\frac{m}{4}$, where $m$ is the infimum of the previous lemma. For $n>1$, let

$$
x_{n}=\bigvee\left\{x \mid G(x) \leq y_{n-1}\right\}=G^{+}\left(y_{n-1}\right) \text { and } y_{n}=G^{r}\left(x_{n}\right)+\frac{m}{4}
$$

Observe that

$$
y_{n}-y_{n-1}=G^{r}\left(x_{n}\right)+\frac{m}{4}-y_{n-1}=G^{r}\left(G^{+}\left(y_{n-1}\right)\right)+\frac{m}{4}-y_{n-1} .
$$

Since $G(x) \leq y_{n-1}$ implies that $x \leq G^{+}\left(y_{n-1}\right)$ it follows that $G(t)>y_{n-1}$ for all $t>G^{+}\left(y_{n-1}\right)$. Therefore,

$$
G^{r}\left(G^{+}\left(y_{n-1}\right)\right) \geq y_{n-1} \text { or } G^{r}\left(G^{+}\left(y_{n-1}\right)\right)-y_{n-1} \geq 0 .
$$

It now follows that

$$
y_{n}-y_{n-1} \geq \frac{m}{4} .
$$

Thus, there is a smallest $N$ such that $y_{N}>G(q)$.

Furthermore, for $n=1, \ldots, N$ we have that $F\left(x_{n}\right)-G^{r}\left(x_{n}\right) \geq m$ which in turn implies that $y_{n}=G^{r}\left(x_{n}\right)+\frac{m}{4}<F\left(x_{n}\right)$. Also, for $1 \leq n<N, G\left(x_{n+1}\right)=G\left(G^{+}\left(y_{n}\right)\right) \leq$ $y_{n}$. Now $G\left(x_{n+1}\right) \leq y_{n}<F\left(x_{n}\right)$ along with $G(q)<y_{N}<F(q)$ implies that $\delta_{x_{n}, y_{n}} \ll F$ for all $n=1, \ldots, N$ and $G(x) \leq \bigvee_{n=1}^{N} \delta_{x_{n}, y_{n}}$. Since « distributes over finite joins, we have that $G \leq \bigvee_{n=1}^{N} \delta_{x_{n}, y_{n}} \ll F$, hence $G \ll F$. 
Looking back to Figure 4.5, observe that as soon as $F$ leaves zero, there is always a separation from $G$ in which it is possible to find another function that is between them. Explicitly, as stated in Theorem 4.10, we can use deltas whose join is a step function that occupies this space between $F$ and $G$. More than that, however, one should see that we can find a function, $H$, between $F$ and $G$ where $H \ll F$ and $G \ll H$. Lemma 4.11 shows that $H \ll F$ exists.

LEMMA 4.11. If $G_{1}, G_{2}, \ldots, G_{n} \ll F$ then $\bigvee G_{i} \ll F$.

Proof. Let $D$ be directed and $\bigvee D \geq F$. Then, for each $i$, there is a $d_{i} \in D$ such that $d_{i} \geq G_{i}$. This implies that

$$
\omega=\bigvee_{i=1}^{n} d_{i} \geq \bigvee_{i=1}^{n} G_{i}
$$

Now, $\omega$ is not necessarily an element in $D$, but because $D$ is directed, there is a $d \in D$ such that $d \geq \omega$.

The information so far should give us a good idea of what the way-below relation is and give some illustration of how two functions are related when one is said to be way-below the other. We also note that in the coming pages, the idea of way-above will be mentioned. Intuitively, we say that if $G$ is way-below $F$ then $F$ is way-above $G$.

The notion of way-above is of more interest since given an arbitrary function, we can always find something way-below. Recall that from Remark 4.3 even the bottom element has something way-below it, namely itself. In the next part where we discuss contraction mapping, it is sometimes more feasible to use way-above in our discussion. 


\section{CHAPTER 5 FUNCTION MAPPINGS WITH RESPECT TO WAY-BELOW}

We have discussed what it means for two residuated functions to be waybelow one another, and we now turn our interest to mappings on spaces of residuated sets and whether or not the object in the range is way-above (or way-below) the input from the domain. That is, given a mapping $\phi: \operatorname{Res}([L, M]) \rightarrow \operatorname{Res}([L, M])$ and $F \in \operatorname{Res}([L, M])$, is $F \ll \phi(F)$ ? As before, we need to restrict ourselves to $L, M$ being real intervals. We focus on the intervals $[p, q],[r, s]$ and consider the functions in $\operatorname{Res}(\operatorname{Res}([0,1]))$, i.e. $\phi: \operatorname{Res}([0,1]) \rightarrow \operatorname{Res}([0,1])$. This allows us to begin work in Probabilistic Metric Spaces where distance distribution functions are in $\operatorname{Res}([0, \infty],[0,1])$.

The results presented began with one basic question, "For which $F \in \operatorname{Res}([p, q])$ and $\phi: \operatorname{Res}([p, q]) \rightarrow \operatorname{Res}([r, s])$ is $F \ll \phi(F)$ ?". That is, can we classify the $\phi$ and $F$ for which $\phi(F)$ maps to an element way above? We also consider under which conditions is the way-below relationship preserved, i.e. if $F$ is way-below $G$ for which $\phi$ is this maintained?

We begin by describing the set of functions that are way above some $F \in$ $\operatorname{Res}([p, q],[r, s])$. What characteristics must a function have to be way-above $F$ ? We will give such a set special notation as follows. Let $\uparrow F$ denote the set of functions that are way above $F$, i.e.

$$
\uparrow F=\{G \in \operatorname{Res}([p, q]) \mid F \ll G\} .
$$

Again, we start with the most simple residuated maps by looking at deltas. 
Since we provided the requirements for a delta to be way below an arbitrary $F$ in the last chapter, the following should be straightforward.

THEOREM 5.1. For $p<a<q$ and $r<b<s$,

$$
\uparrow \delta_{a, b}=\left\{F \in \operatorname{Res}([p, q],[r, s]) \mid a>b_{F} \& F(a)>b\right\} .
$$

Proof. This is an immediate consequence of Theorem 4.8.

After establishing such a set can exist, we would like to show that it is nonempty and not trivial for functions other than deltas. The following gives us the requirement on $F$ for which there are functions way-above it, or $\uparrow F \neq \varnothing$. Using the information previously discussed, a residuated map has at least one other function above it as long as it does not jump at zero and it never attains the top value (in this case, $s$ ).

THEOREM 5.2. The set of functions that are way above $F \in \operatorname{Res}([p, q],[r, s])$ is nonempty if and only if $b_{F}>0$ and $F(q)<s$.

Proof. Let $F \in \operatorname{Res}([p, q],[r, s])$. Suppose $\uparrow F \neq \varnothing$, that is, there exists $G \in$ $\operatorname{Res}([p, q],[r, s])$ such that $F \ll G$. Suppose $b_{F} \ngtr 0$. Since $G \geq F$, consider the directed set, D, consisting of

$$
d_{n}(x)= \begin{cases}0 & x \leq \frac{1}{n} \\ G(x) & \text { otherwise }\end{cases}
$$

Then, $\vee D=G$, however, $F \nless d$ for all $d \in D$. Thus, $F \nless k$.

Now suppose $F(q)=s$ and $b_{F}>0$. Let $K=\bigwedge\{x \mid G(x)=s\}$, then for $x<K$, $G(x)<s$ and define

$$
d_{n}(x)=\left\{\begin{array}{cc}
G(x) & \text { for } x \leq K-\frac{1}{n} \\
G\left(K-\frac{1}{n}\right) & \text { for } x>K-\frac{1}{n}
\end{array}\right.
$$


Then, since $G(x) \geq F(x)$ for all $x \in[p, q]$ and $F(q)=s$, we have $d_{n} \| F$ but $\vee d_{n}=G \geq F$. Since the $d_{n}$ form a directed set, we have $F \ll G$.

Therefore $\uparrow F \neq \varnothing$ implies $b_{F}>0$ and $F(q)<s$.

In the other direction, consider $b_{F}>p$ and $F(q)<s$. Let $G$ be the top function in $\operatorname{Res}([p, q],[r, s])$, that is, $G=\delta_{p, s}$ where $G(p)=p$ and $G(x)=s$ for $x \neq p$. Consider

$$
H=\delta_{\frac{b_{F}+p}{2}}, \frac{F(q)+s}{2} .
$$

By Theorem 4.10, $F \ll G$ because $F \leq H$ and $H \ll G$.

We begin our observations with some strong restrictions by first looking at order automorphisms. It turns out that an order isomorphism will preserve the way-below relationship.

THEOREM 5.3. Let $\phi: \operatorname{Res}\left(L_{1}, M_{1}\right) \rightarrow \operatorname{Res}\left(L_{2}, M_{2}\right)$ be an order isomorphism, then $F \ll G$ if and only if $\phi(F) \ll \phi(G)$.

Proof. Let $\phi$ an order isomorphism and $D \subseteq \operatorname{Res}(L, M)$ be a directed set with $\vee D \geq \phi(G)$. Let $\widetilde{D}=\left\{\phi^{-1}(d) \mid d \in D\right\}$. Then, $\widetilde{D}$ is a directed set since both $\phi$ and $\phi^{-1}$ preserve order.

Observe,

$$
\bigvee \widetilde{D}=\bigvee\left\{\phi^{-1}(d) \mid d \in D\right\}=\phi^{-1}(\bigvee D) \geq \phi^{-1}(\phi(G))=G
$$

This means $\bigvee \widetilde{D} \geq G$. Since $F \ll G$, there exists $\tilde{d} \in \widetilde{D}$ such that $F \leq \tilde{d}$. This implies,

$$
\phi(F) \leq \phi(\tilde{d}) \in D
$$

Thus,

$$
\phi(F) \ll \phi(G) .
$$

The converse is analogous. 
In 1988, R.C. Powers published a classification theorem for order automorphisms $([7])$. According to Powers, every order automorphism in Res $(L)$ is either Type 1 or Type 2 .

THEOREM 5.4. (Powers $1988[$ [7]) The map $\phi$ belongs to Aut $(\operatorname{Res}([p, q],[r, s]))$ if and only if for all $F$ in $\operatorname{Res}([p, q],[r, s])$, one of the following holds:

(i) $\phi(F)=\theta \circ F \circ \tau$ where $\theta \in \operatorname{Aut}[r, s]$ and $\tau \in \operatorname{Aut}[p, q]$ ( Type 1 );

(ii) $\phi(F)=\alpha \circ F^{+} \circ \beta$ where $\alpha$ and $\beta$ are dual automorphisms from $[p, q]$ onto $[r, s]$ (Type 2) and $F^{+}$is the associated residual of $F$.

Type 1 automorphisms are very nice in that under certain circumstances they can map way-below (or way-above). Because Type 1 is a composition with continuous increasing bijections, we will need that $F$ cannot reach 1 and $b_{F} \neq 0$.

THEOREM 5.5. Let $\phi$ be an order automorphism of Res $([p, q],[r, s])$ such that $\phi(F)=\theta \circ F \circ \gamma$ where $\gamma:[p, q] \rightarrow[p, q], \theta:[r, s] \rightarrow[r, s]$, are both order automorphisms with the property that $\gamma(x)<x$ for all $x \in(p, q)$ and $\theta(x)<x$ for all $x \in(r, s)$. If $b_{F}>p$ and $F(q)<s$, then $\phi(F) \ll F$.

Proof. If $F=0_{F}$, then $\phi\left(0_{F}\right)=0_{F} \ll 0_{F}=0$ for all $x$.

Otherwise, using the properties of $\theta$ and $\gamma$, we get

$$
(\phi(F))(x)=\theta(F(\gamma(x))) \leq F(\gamma(x))
$$

Since $F$ is nondecreasing, we get

$$
F(\gamma(x)) \leq F(x)
$$

Thus,

$$
\phi(F) \leq F \text { for all } F \in \operatorname{Res}([p, q],[r, s]) .
$$


Since $\theta$ is a bijection, we have $\theta(u)=r \Longleftrightarrow u=\theta^{-1}(r)=r$, thus

$$
(\phi(F))(x)=\theta(F(\gamma(x)))=r \Longleftrightarrow F(\gamma(x))=r .
$$

With $b_{F}=\bigvee\{x \mid F(x)=r\}>p$, we get

$$
\begin{aligned}
b_{\phi(F)} & =\bigvee\{x \mid(\phi(F))(x)=r\} \\
& =\bigvee\{x \mid F(\gamma(x))=r\} \quad \text { Let } y=\gamma(x) \text {, then } \gamma^{-1}(y)=x \\
& =\bigvee\left\{\gamma^{-1}(y) \mid F(y)=r\right\} \\
& =\gamma^{-1}(\bigvee\{y \mid F(y)=r\}) \text { since } \gamma^{-1} \text { is an order automorphism. } \\
& =\gamma^{-1}\left(b_{F}\right)
\end{aligned}
$$

Thus,

$$
\phi(F)(x)=r \Longleftrightarrow x \leq \gamma^{-1}\left(b_{F}\right) \Longleftrightarrow \gamma(x) \leq b_{F} .
$$

Since $\gamma(x)<x$, we have $\gamma^{-1}(x)>x$ for $x \in[p, q]$. Hence,

$$
b_{\phi(F)}>b_{F}
$$

We require that $b_{F}>0$ since

$$
\theta(F(\gamma(0)))=0 \text { if } b_{F}=0 \text { then } b_{\phi(F)}=0
$$

We also observe $\phi(F)(q)<F(q)$ since

$$
\theta(F(\gamma(q)))=\theta(F(q))<F(q)
$$

Similarly we need $F(q)<s$ since if

$$
F(q)=s \text { then } \theta(F(\gamma(q)))=s \text { is possible. }
$$

Now,

$$
\lim _{x \rightarrow a^{+}} \phi(F)(x)<F(a) .
$$


Then,

$$
\lim _{n \rightarrow \infty} \theta\left(F\left(\gamma\left(a+\frac{1}{n}\right)\right)\right)=\theta\left(\lim _{n \rightarrow \infty} F\left(\gamma\left(a+\frac{1}{n}\right)\right)\right) .
$$

And for $n$ large, $\gamma\left(a+\frac{1}{n}\right)<a$. Then,

$$
F\left(\gamma\left(a+\frac{1}{n}\right)\right) \leq F(a) \leq \theta(F(a))<F(a)
$$

Therefore, when $b_{F}>0$ and $F(q)<s$, by Theorem 4.10 , we have $\phi(F) \ll F$.

This also easily establishes that we can have a $\phi$ of Type 1 that maps wayabove for which we provide the following corollary. The proof is analogous taking into consideration the changes to $\theta$ and $\gamma$.

Corollary 5.6. Let $\phi$ be an order automorphism of Res $([p, q],[r, s])$ such that $\phi(F)=$ $\theta \circ F \circ \gamma$ where $\gamma:[p, q] \rightarrow[p, q], \theta:[r, s] \rightarrow[r, s]$, are both order automorphisms with the property that $\gamma(x)>x$ for all $x \in[p, q]$ and $\theta(x)>x$ for all $x \in[r, s]$. Then, $F \ll \phi(F)$ for all $F$ such that $\uparrow F \neq \varnothing$.

On the other hand, Type 2 order automorphisms do not map with a waybelow relationship. In fact, if $\phi$ is an order automorphism of Type 2, not only can we always find a $\delta_{a, b}$ for which $\phi\left(\delta_{a, b}\right) \nless \delta_{a, b}$, but we also know that $\delta_{a, b}$ and $\phi\left(\delta_{a, b}\right)$ are non-comparable when $\delta_{a, b} \neq \delta_{p, r}=0$.

THEOREM 5.7. If $\phi(F)=\alpha \circ F^{+} \circ \beta$, then there exists $\delta_{a, b}$, with $a \in(p, q], b \in(r, s]$ such that $\phi\left(\delta_{a, b}\right) \| \delta_{a, b}$ and $\phi\left(\delta_{a, b}\right) \nless \delta_{a, b}$. Note: $\phi\left(\delta_{a, b}\right)=\delta_{\beta^{-1}(b), \alpha(a)}$

Proof. Since $\alpha$ and $\beta$ are bijections and therefore invertible, choose any $t \in(r, s)$ which means

$$
\beta^{-1}(t)<q \quad \text { and } \quad \gamma^{-1}(t)<q
$$

So, there exists an $a \in(p, q)$ such that,

$$
a>\beta^{-1}(t)
$$


and

$$
a>\alpha^{-1}(t)
$$

Applying the strictly decreasing function, $\alpha$, to both sides of (5.2), we get

$$
\alpha(a)<t
$$

This implies,

$$
\begin{array}{r}
\delta_{\beta^{-1}(t), \alpha(a)} \| \delta_{a, t} \\
\phi\left(\delta_{a, t}\right) \| \delta_{a, t}
\end{array}
$$

And, in particular, $\phi\left(\delta_{a, t}\right) \nless \delta_{a, t}$

Order Automorphisms were a place to start looking but a bit too restrictive for our purposes and application. Thus, we turn our attention to mappings on $\operatorname{Res}([p, q],[r, s])$ in general. As discussed before, we first look at how such a mapping, $\phi$, behaves with deltas and extend our findings from there.

The first step is to look at $\phi$ that are induced similar to Type 1 order automorphisms. If applying $\phi$ to $\delta_{a, b}$ results in a delta whose first index is just a function of the original $a$ and whose second index is just a function of the original $b$ (i.e. $\left.\phi\left(\delta_{a, b}\right)=\delta_{\tau(a), \theta(b)}\right)$, we can identify the monotonicity and continuity of those functions $(\tau, \theta)$.

More formally, for residuated $\phi: \operatorname{Res}([p, q],[r, s]) \rightarrow \operatorname{Res}([p, q],[r, s])$ and $\phi\left(\delta_{a, b}\right)=\delta_{\tau(a), \theta(b)}$, then $\tau$ is increasing and right continuous while $\theta$ is increasing and left continuous.

LEMMA 5.8. Suppose $\phi: \operatorname{Res}([p, q],[r, s]) \rightarrow \operatorname{Res}([p, q],[r, s])$ is residuated and

$$
\phi\left(\delta_{a, b}\right)=\delta_{\tau(a), \theta(b)} \text { for all } a \in[p, q] \text { and } b \in[r, s] \text {. }
$$

Then, $\tau:[p, q] \rightarrow[p, q]$ and $\theta:[r, s] \rightarrow[r, s]$ are nondecreasing. 
Proof. If $\phi$ is residuated, then $\phi$ is nondecreasing and maps $\delta_{p, r} \rightarrow \delta_{p, r}$. Suppose $\phi \not \equiv \delta_{p, r}$. Thus, there exists an $b \in(r, s]$ such that $\theta(b) \neq r$. Now, suppose $b_{1}<b_{2}$ in $(\mathrm{r}, \mathrm{s}]$.

Then, $\delta_{a, b_{1}}<\delta_{a, b_{2}}$ which implies $\phi\left(\delta_{a, b_{1}}\right) \leq \phi\left(\delta_{a, b_{2}}\right)$. Thus, $\delta_{\tau(a), \theta\left(b_{1}\right)} \leq \delta_{\tau(a), \theta\left(b_{2}\right)}$.

Therefore,

$$
\theta\left(b_{1}\right) \leq \theta\left(b_{2}\right)
$$

Similarly, there is an $a \in[p, q)$ such that $\tau(a) \neq q$, and we suppose $a_{1}<a_{2}$ in $[\mathrm{p}, \mathrm{q})$.

Then, $\delta_{a_{2}, b}<\delta_{a_{1}, b}$ which implies $\phi\left(\delta_{a_{2}, b}\right) \leq \phi\left(\delta_{a_{1}, b}\right)$. Thus, $\delta_{\tau\left(a_{2}\right), \theta(b)} \leq \delta_{\tau\left(a_{1}\right), \theta(b)}$.

Therefore,

$$
\tau\left(a_{1}\right) \leq \tau\left(a_{2}\right)
$$

LEMMA 5.9. Let $\phi: \operatorname{Res}([p, q],[r, s]) \rightarrow \operatorname{Res}([p, q],[r, s])$ be residuated and $\phi\left(\delta_{a, b}\right)=$ $\delta_{\tau(a), \theta(b)}$. Then $\theta:[r, s] \rightarrow[r, s]$ is left continuous and $\tau:[p, q] \rightarrow[p, q]$ is right continuous.

Proof. Without loss of generality, assume $\phi \not \equiv \delta_{p, r}$ and let $a<q, b>r$. Choose $b$ such that $\theta(b)>r$ and $b$ such that $\tau(a)<q$ then consider,

$$
\delta_{a, b}=\bigvee_{t<b} \delta_{a, t}=\lim _{t \rightarrow b^{-}} \delta_{a, t}=\delta_{a, \lim _{t \rightarrow b^{-}} t}
$$

Since $\phi$ is residuated,

$$
\begin{aligned}
\delta_{\tau(a), \theta(b)}=\phi\left(\delta_{a, b}\right) & =\phi\left(\bigvee_{t<b} \delta_{a, t}\right) \\
& =\bigvee_{t<b} \phi\left(\delta_{a, t}\right) \\
& =\bigvee_{t<b} \delta_{\tau(a), \theta(t)} \\
& =\lim _{t \rightarrow b^{-}} \delta_{\tau(a), \theta(t)} \\
& =\delta_{\tau(a), \theta\left(\lim _{t \rightarrow b^{-}} t\right)}
\end{aligned}
$$


Therefore, $\theta$ must be left continuous.

Again without loss of generality assume $a<q, b>r$. Now consider,

$$
\delta_{a, b}=\bigvee_{s>a} \delta_{s, b}=\lim _{s \rightarrow a^{+}} \delta_{s, b}=\delta_{s \rightarrow a^{+}} s, b
$$

If $\phi$ is residuated,

$$
\begin{aligned}
\delta_{\tau(s), \theta(b)}=\phi\left(\delta_{a, b}\right) & =\phi\left(\bigvee_{s>a} \delta_{s, b}\right) \\
& =\bigvee_{s>a} \phi\left(\delta_{s, b}\right) \\
& =\bigvee_{s>a} \delta_{\tau(s), \theta(b)} \\
& =\lim _{s \rightarrow a^{+}} \delta_{\tau(s), \theta(b)} \\
& =\delta_{\tau\left(\lim _{s \rightarrow a^{+}} s\right), \theta(b)}
\end{aligned}
$$

Therefore, $\tau$ must be right continuous.

Now, we consider what happens if applying $\phi$ to $\delta_{a, b}$ results in a delta where the indices are functions of the opposite index from the input, i.e. similar to Type 2 order automorphisms. Thus, if

$$
\phi\left(\delta_{a, b}\right)=\delta_{\alpha(b), \beta(a)}
$$

for some sets

$$
\begin{aligned}
& \alpha:[r, s] \rightarrow[p, q] \\
& \beta:[p, q] \rightarrow[r, s]
\end{aligned}
$$

we have that the functions $\alpha, \beta$ are both decreasing while $\alpha$ is left continuous and $\beta$ is right continuous.

LEMMA 5.10. Suppose $\phi: \operatorname{Res}([p, q],[r, s]) \rightarrow \operatorname{Res}([p, q],[r, s])$ is residuated and

$$
\phi\left(\delta_{a, b}\right)=\delta_{\alpha(b), \beta(a)} \text { for all } a \in[p, q] \text { and } b \in[r, s]
$$

Then, $\alpha:[r, s] \rightarrow[p, q]$ and $\beta:[p, q] \rightarrow[r, s]$ are decreasing and $\alpha(r)=q, \beta(q)=r$. 
Proof. If $\phi$ is residuated, then $\phi$ is nondecreasing and maps $\delta_{p, r} \rightarrow \delta_{p, r}$. Suppose $\phi \not \equiv \delta_{p, r}$. Thus, there exists an $a \in(p, q)$ such that $\beta(a) \neq r$. Now, suppose $b_{1}<b_{2}$ in $(\mathrm{r}, \mathrm{s})$.

Then, $\delta_{a, b_{1}}<\delta_{a, b_{2}}$ which implies $\phi\left(\delta_{a, b_{1}}\right) \leq \phi\left(\delta_{a, b_{2}}\right)$. Thus, $\delta_{\alpha\left(b_{1}\right), \beta(a)} \leq \delta_{\alpha\left(b_{2}\right), \beta(a)}$. Therefore,

$$
\alpha\left(b_{1}\right) \geq \alpha\left(b_{2}\right)
$$

Similarly, there is a $b \in(r, s)$ such that $\alpha(b) \neq p$, and we suppose $a_{1}<a_{2}$ in (p,q).

Then, $\delta_{a_{2}, b}<\delta_{a_{1}, b}$ which implies $\phi\left(\delta_{a_{2}, b}\right) \leq \phi\left(\delta_{a_{1}, b}\right)$. Thus, $\delta_{\alpha(b), \beta\left(a_{2}\right)} \leq \delta_{\alpha(b), \beta\left(a_{1}\right)}$. Therefore,

$$
\beta\left(a_{1}\right) \geq \beta\left(a_{2}\right)
$$

We have

$$
\delta_{p, r}=\delta_{a, r}=\delta_{q, a} \text { for all } a
$$

Thus,

$$
\delta_{\alpha(r), \beta(a)}=\delta_{q, \beta(a)} \text { for all } a
$$

and

$$
\delta_{\alpha(a), \beta(q)}=\delta_{a, r} \text { for all } a \text {. }
$$

This implies that

$$
\beta(q)=r \text { and } \alpha(r)=q
$$

LEMMA 5.11. Let $\phi\left(\delta_{a, b}\right)=\delta_{\alpha(b), \beta(a)}$ then $\phi: \operatorname{Res}([p, q],[r, s]) \rightarrow \operatorname{Res}([p, q],[r, s])$ is residuated if $\alpha$ is left continuous and $\beta$ is right continuous.

Proof. Without loss of generality, assume $\phi \not \equiv \delta_{p, r}$ and $a<q, b>r$. Choose $a$ such that $\beta(a) \neq r$ and consider,

$$
\delta_{a, b}=\bigvee_{t<b} \delta_{a, t}=\lim _{t \rightarrow b^{-}} \delta_{a, t}=\delta_{a, \lim _{t \rightarrow b^{-}}} t
$$


Since $\phi$ is residuated,

$$
\begin{aligned}
\delta_{\alpha(b), \beta(a)}=\phi\left(\delta_{a, b}\right) & =\phi\left(\bigvee_{t<b} \delta_{a, t}\right) \\
& =\bigvee_{t<b} \phi\left(\delta_{a, t}\right) \\
& =\bigvee_{t<b} \delta_{\alpha(t), \beta(a)} \\
& =\lim _{t \rightarrow b^{-}} \delta_{\alpha(t), \beta(a)} \\
& =\delta_{\alpha\left(\lim _{t \rightarrow b^{-}} t\right), \beta(a)}
\end{aligned}
$$

Therefore, $\alpha$ must be left continuous.

Again without loss of generality assume $a<q, b>r$. Now consider,

$$
\delta_{a, b}=\bigvee_{s>a} \delta_{s, b}=\lim _{s \rightarrow a^{+}} \delta_{s, b}=\delta_{\lim _{s \rightarrow a^{+}} s, b}
$$

If $\phi$ is residuated,

$$
\begin{aligned}
\delta_{\alpha(b), \beta(a)}=\phi\left(\delta_{a, b}\right) & =\phi\left(\bigvee_{s>a} \delta_{s, b}\right) \\
& =\bigvee_{s>a} \phi\left(\delta_{s, b}\right) \\
& =\bigvee_{s>a} \delta_{\alpha(b), \beta(s)} \\
& =\lim _{s \rightarrow a^{+}} \delta_{\alpha(b), \beta(s)} \\
& =\delta_{\alpha(b), \beta\left(\lim _{s \rightarrow a^{+}} s\right)}
\end{aligned}
$$

Therefore, $\beta$ must be right continuous.

Next we consider more general mappings: the case where applying $\phi$ to $\delta_{a, b}$ results in a delta whose indices are functions of two variables and dependent on both $a$ and $b$ from the input. Such a $\phi$ is given by

$$
\phi\left(\delta_{a, b}\right)=\delta_{\gamma(a, b), \sigma(a, b)}
$$


and we get that $\gamma$ is increasing in the first variable, decreasing in the second and $\sigma$ is increasing the second variable, decreasing in the first. Both $\gamma$ and $\sigma$ are left continuous in second variable and right continuous in the first variable.

LEMMA 5.12. Suppose $\phi: \operatorname{Res}([p, q],[r, s]) \rightarrow \operatorname{Res}([p, q],[r, s])$ is residuated and

$$
\phi\left(\delta_{a, b}\right)=\delta_{\gamma(a, b), \sigma(a, b)} \text { for all } a \in[p, q] \text { and } b \in[r, s]
$$

Then, $\gamma:[p, q] \times[r, s] \rightarrow[p, q]$ and $\sigma:[p, q] \times[r, s] \rightarrow[r, s]$ must be decreasing in $b$ and $a$, respectively, and $\gamma, \sigma$ are increasing in $a$ and $b$, respectively.

Proof. Since $\phi$ is residuated, then $\phi$ is increasing. Let $a_{1}, a_{2} \in[p, q]$ and $b_{1}, b_{2} \in[r, s]$. Assume $a_{1} \geq a_{2}$ and $b_{1} \leq b_{2}$.

Then,

$$
\begin{gathered}
\delta_{a_{1}, b_{1}} \leq \delta_{a_{2}, b_{2}} \\
\phi\left(\delta_{a_{1}, b_{1}}\right) \leq \phi\left(\delta_{a_{2}, b_{2}}\right) \\
\delta_{\gamma\left(a_{1}, b_{1}\right), \sigma\left(a_{1}, b_{1}\right)} \leq \delta_{\gamma\left(a_{2}, b_{2}\right), \sigma\left(a_{2}, b_{2}\right)}
\end{gathered}
$$

Thus,

$$
\gamma\left(a_{1}, b_{1}\right) \geq \gamma\left(a_{2}, b_{2}\right) \text { and } \sigma\left(a_{1}, b_{1}\right) \leq \sigma\left(a_{2}, b_{2}\right)
$$

Now, if $b_{1}=b_{2}$,

$\gamma$ is increasing in $a$ and $\sigma$ is decreasing in $a$

And if $a_{1}=a_{2}$,

$\gamma$ is decreasing in $b$ and $\sigma$ is increasing in $b$

LEMMA 5.13. Let $\phi\left(\delta_{a, b}\right)=\delta_{\gamma(a, b), \sigma(a, b)}$ then $\phi \in \operatorname{Res}([p, q],[r, s])$ if $\gamma:[p, q] \times$ $[r, s] \rightarrow[p, q]$ and $\sigma:[p, q] \times[r, s] \rightarrow[r, s]$ are left continuous in the second variable and right continuous in the first variable. 
Proof. Suppose $\phi: \operatorname{Res}([p, q],[r, s]) \rightarrow \operatorname{Res}([p, q],[r, s])$ is residuated and

$$
\phi\left(\delta_{a, b}\right)=\delta_{\gamma(a, b), \sigma(a, b)} \text { for all } a \in[p, q] \text { and } b \in[r, s] \text {. }
$$

Without loss of generality, assume $\phi \not \equiv \delta_{p, r}$ and $a<q, b>r$, then we can choose $a$ such that $\gamma(a, b) \neq q$ and $\sigma(a, b) \neq r$ and consider,

$$
\delta_{a, b}=\bigvee_{t<b} \delta_{a, t}=\lim _{t \rightarrow b^{-}} \delta_{a, t}=\delta_{a, \lim _{t \rightarrow b^{-}}} t
$$

If $\phi$ is residuated,

$$
\begin{aligned}
\delta_{\gamma(a, b), \sigma(a, b)}=\phi\left(\delta_{a, b}\right) & =\phi\left(\bigvee_{t<b} \delta_{a, t}\right) \\
& =\bigvee_{t<b} \phi\left(\delta_{a, t}\right) \\
& =\bigvee_{t<b} \delta_{\gamma(a, t), \sigma(a, t)} \\
& =\lim _{t \rightarrow b^{-}} \delta_{\gamma(a, t), \sigma(a, t)} \\
& =\delta_{\gamma\left(a, \lim _{t \rightarrow b^{-}} t\right), \sigma\left(a, \lim _{t \rightarrow b^{-}} t\right)}
\end{aligned}
$$

Therefore, $\gamma, \sigma$ must be left continuous in the second variable.

Similarly, we now consider,

$$
\delta_{a, b}=\bigvee_{u>a} \delta_{u, b}=\lim _{s \rightarrow a^{+}} \delta_{u, b}=\delta_{\lim _{u \rightarrow a^{+}} u, b}
$$

If $\phi$ is residuated,

$$
\begin{aligned}
\delta_{\gamma(a, b), \sigma(a, b)}=\phi\left(\delta_{a, b}\right) & =\phi\left(\bigvee_{u>a} \delta_{u, b}\right) \\
& =\bigvee_{u>a} \phi\left(\delta_{u, b}\right) \\
& =\bigvee_{u>a} \delta_{\gamma(a, b), \sigma(a, b)} \\
& =\lim _{u \rightarrow a^{+}} \delta_{\gamma(a, b), \sigma(a, b)} \\
& =\delta_{\gamma\left(\lim _{u \rightarrow a^{+}} u, b\right), \sigma\left(\lim _{u \rightarrow a^{+}} u, b\right)}
\end{aligned}
$$

Therefore, $\gamma, \sigma$ must be right continuous in the first variable. 
These lemmas tell us a lot about what happens when a residuated map, $\phi$, maps a delta to another delta. In fact, this gives us enough information to know that iterations of $\phi$ will eventually map to the top element, $\delta_{q, s}$.

THEOREM 5.14. Let $\phi: \operatorname{Res}([p, q],[r, s]) \rightarrow \operatorname{Res}([p, q],[r, s])$ and be residuated. Suppose $\phi$ maps deltas to deltas, then for all $F \neq \delta_{0,0}$ with $\uparrow F \neq \varnothing$, we have

$$
F \ll \phi(F) \Longrightarrow \lim _{n \rightarrow \infty} \phi^{n}(F)=\delta_{0,1}
$$

Proof. Since $\uparrow \delta_{p, b}=\varnothing$ for $b \neq r$ and $\uparrow \delta_{a, s}=\varnothing$ for $a \neq q$, we may assume

$$
a>p \text { and } b<s .
$$

Case 1: Type 1 mappings of the form $\theta \circ \delta_{a, b} \circ \tau^{-1}=\phi\left(\delta_{a, b}\right)=\delta_{\tau(a), \theta(b)}$

Suppose $a>0, b<1$ and $\lim _{n \rightarrow \infty} \phi^{n}\left(\delta_{a, b}\right) \neq \delta_{0,1}$. Since $\phi^{n}\left(\delta_{a, b}\right)=\delta_{\tau^{n}(a), \theta^{n}(b)}$, we have $\lim _{n \rightarrow \infty} \theta^{n}(b)=L_{1}<1$ or $\lim _{n \rightarrow \infty} \tau^{n}(a)=L_{2}>0$.

Suppose $\lim _{n \rightarrow \infty} \theta^{n}(b)=L_{1}<1$. Then consider $\delta_{a, L_{1}}$ and note that $\uparrow \delta_{a, L_{1}} \neq \varnothing$. Since $\lim _{n \rightarrow \infty} \theta^{n}(b)=L_{1}<1$, we get

$$
\begin{aligned}
& \phi\left(\delta_{a, L_{1}}\right)=\theta \circ \delta_{a, L_{1}} \circ \tau^{-1}=\delta_{\tau(a), \theta\left(L_{1}\right)}=\delta_{\tau(a), L_{1}} \\
L_{1}=\lim _{n \rightarrow \infty} \theta^{n}(b)= & \lim _{n \rightarrow \infty} \theta^{n+1}(b) \\
= & \theta\left(\lim _{n \rightarrow \infty} \theta^{n}(b)\right) \text { since } \theta \text { has to be left continuous by Lemma 5.9. } \\
= & \theta\left(L_{1}\right)
\end{aligned}
$$

Now by Lemma 5.8,

$$
\delta_{a, L_{1}} \ll \phi\left(\delta_{a, L_{1}}\right)=\delta_{\tau(a), \theta\left(L_{1}\right)}=\delta_{\tau(a), L_{1}}
$$

This is a contradiction, $\delta_{a, L_{1}} \nless \phi\left(\delta_{a, L_{1}}\right)$ and so,

$$
\lim _{n \rightarrow \infty} \theta^{n}(b)=L_{1}
$$


Now, suppose $\lim _{n \rightarrow \infty} \tau^{n}(a)=L_{2}>0$. Consider $\delta_{L_{2}, b}$ and note that $\uparrow \delta_{L_{2}, b} \neq \varnothing$. If $\delta_{L_{2}, b} \ll \phi\left(\delta_{L_{2}, b}\right)=\delta_{\tau\left(L_{2}\right), \theta(b)}$, this would require $L_{2}<\tau\left(L_{2}\right)$. However,

$$
\begin{aligned}
L_{2}=\lim _{n \rightarrow \infty} \tau^{n}(b) & =\lim _{n \rightarrow \infty} \tau^{n+1}(b) \\
& =\tau\left(\lim _{n \rightarrow \infty} \tau^{n}(b)\right) \text { since } \tau \text { is right continuous by Lemma } 5.9 \\
& =\tau\left(L_{2}\right)
\end{aligned}
$$

contradicting $\delta_{L_{2}, b} \ll \delta_{\tau\left(L_{2}\right), b}=\phi\left(L_{2}, b\right)$. Thus,

$$
\lim _{n \rightarrow \infty} \tau^{n}(b)=0
$$

Case 2: Type 2 mappings of the form $\alpha \circ \delta_{a, b}^{+} \circ \beta^{-1}=\phi\left(\delta_{a, b}\right)=\delta_{\alpha(b), \beta(a)}$

According to Theorem 4.7, $\delta_{a, b} \ll \delta_{\alpha(b), \beta(a)}$ if and only if $\alpha(b)<a$ and $\beta(a)>b$.

So, suppose $\alpha(b)<a$ and $\beta(a)>b$. Fix $a \in(0,1)$.

We note,

$$
\lim _{b \rightarrow 1} \delta_{a, b}=\delta_{a, 1}
$$

And,

$$
\phi\left(\lim _{b \rightarrow 1} \delta_{a, b}\right)=\delta_{\alpha\left(\lim _{b \rightarrow 1} b\right), \beta(a)}
$$

As $b$ goes to $1, \beta(a)$ must go to 1 for all $a$ since $\beta(a)>b$.

Similarly, fixing $b \in(0,1)$, we get that as $a$ goes to $0, \alpha(b)$ must also go to zero since $\alpha(b)<a$. Thus,

$$
\phi\left(\delta_{a, b}\right)=\delta_{0,1}
$$

Case $3: \phi\left(\delta_{a, b}\right)=\delta_{\gamma(a, b), \sigma(a, b)}$

Similar to case 2 , we need only consider $\gamma(a, b)<a$ and $\sigma(a, b)>b$. Fix $a \in(0,1)$. As noted before,

$$
\lim _{b \rightarrow 1} \delta_{a, b}=\delta_{a, 1}
$$


And,

$$
\phi\left(\lim _{b \rightarrow 1} \delta_{a, b}\right)=\delta_{\gamma\left(a, \lim _{b \rightarrow 1} b\right), \sigma\left(a, \lim _{b \rightarrow 1} b\right)}
$$

As $b$ goes to 1 , then $\sigma(a, b)$ is forced to 1 because $\sigma(a, b)>b$. Similarly, fixing $b \in(0,1)$, we get that as $a$ goes to $0, \gamma(a, b)$ must also go to zero since $\gamma(a, b)<a$. Again resulting in,

$$
\phi\left(\delta_{a, b}\right)=\delta_{0,1}
$$

Now, let $F \in \Delta^{+} \backslash\left\{\delta_{0,0}\right\}$. Then, there is an $x_{0} \in(0,1)$ such that $F\left(x_{0}\right)>0$. Then,

$$
\delta_{x_{0}, \frac{1}{2} F\left(x_{0}\right)} \leq F
$$

Apply $\phi^{n}$ to both sides,

$$
\phi^{n}\left(\delta_{x_{0}, \frac{1}{2} F\left(x_{0}\right)}\right) \leq \phi^{n}(F)
$$

Taking the limit as $n$ goes to infinity, we get

$$
\delta_{0,1}=\lim _{n \rightarrow \infty} \phi^{n}\left(\delta_{x_{0}, \frac{1}{2} F\left(x_{0}\right)}\right) \leq \lim _{n \rightarrow \infty} \phi^{n}(F)
$$

Therefore,

$$
\lim _{n \rightarrow \infty} \phi^{n}(F)=\delta_{0,1}
$$

The result that successive iterations of $\phi$ gives us a fixed point, namely the top element $\delta_{0,1}$ is what we are hoping for, but the requirement that $\phi$ maps deltas to deltas is quite restrictive. So, we would like to extend this same result to any nondecreasing $\phi$ in our space.

We then considered a less restrictive case and tried to relax the condition that $\phi$ be residuated. We conclude that even requiring $\phi$ to be monotone, if $\phi$ is not residuated, then $\phi^{n}(F)$ does not necessarily go to $\delta_{0,1}$ as illustrated by the following counterexample: 
EXAMPLE 5.15. Consider $\phi: \operatorname{Res}([p, q],[r, s]) \rightarrow \operatorname{Res}([p, q],[r, s])$, a monotone function, and fix $a \in(0,1)$ :

$$
\phi(F)= \begin{cases}\delta_{\frac{a+b_{F}}{2}, \frac{1+F(1)}{2}} & \text { if } F \ll \delta_{a, 1} \\ \delta_{0,1} & \text { if } F \ll \delta_{a, 1}\end{cases}
$$

Clearly $\phi\left(\delta_{a, b}\right) \gg \delta_{a, b}$ for all $a, b \in(0,1)$. Furthermore, if $F \ll \delta_{a, 1}$, then $\phi^{n}(F) \rightarrow \delta_{a, 1}$ as $n \rightarrow \infty$, but since $\phi$ is not residuated, we do not get that $\delta_{a, 1}$ has to be a fixed point. Thus dropping the requirement that $\phi$ be residuated (even at just one point) no longer allows the conclusion of Theorem 5.14. 


\section{CHAPTER 6 \\ INTRODUCTION TO PROBABILISTIC METRIC SPACES AND CONTRACTION MAPPINGS}

The research presented regarding the way-below relation has application in probabilistic metric spaces. In this chapter, we will introduce probabilistic metric spaces (or PM spaces) and contraction mappings. We present some basic definitions below which can be found in the book Probabilistic Metric Spaces by B. Schweizer and A. Sklar [11] or references therein.

The general idea of a probabilistic metric space is that in reality we cannot measure distances accurately but that a series of measurements will give us a likelihood of distances. Thus, a probabilistic metric space is a set of points where distances are measured by so called distance distribution functions (d.d.f.). The interpretation will be that such a d.d.f. $F(x)$ gives the probability that the distance of two points is less that or equal to $x$. Thus, we begin with this definition because we will need it.

DEFINITION 6.1 (distribution function, distance distribution function). A distribution function (d.f.) is a nondecreasing function, $F$ defined on the extended nonnegative reals, $[0, \infty]$, with $F(0)=0$ and $F(\infty)=1$. We further assume that $F$ is left continuous on $(0, \infty)$. We denote the space of all such functions by $\Delta^{+}$.

We note that $\Delta^{+}$does not quite fit with our notion of residuated mappings as defined in Chapter 3. For that to work, we need $F:[0, \infty] \rightarrow[0,1]$ to satisfy $F(0)=0, F$ nondecreasing on $[0, \infty]$, and left continuous on $(0, \infty]$. However, $\Delta^{+}$is needed for the probability interpretation of the d.d.f. that $F(\infty)=1$. Fortunately, 
it is easy to see that $\Delta^{+} \cong \operatorname{Res}([0, \infty],[0,1])$ via $F \in \Delta^{+} \rightarrow \widetilde{F} \in \operatorname{Res}([0, \infty],[0,1])$ with $F(x)=\widetilde{F}(x)$ for all $x$ in $[0, \infty)$. This is clearly bijective and order preserving with an order preserving inverse and thus an automorphism.

Now that the usual metric of $[0, \infty)$ is replaced by the d.d.f.'s in $\Delta^{+}$, we need to generalize the properties of a metric space to this setting. Recall that $(X, d)$ is a metric space if

i.) $d(x, y)=0$ if and only if $x=y$,

ii.) $d(x, y)=d(y, x)$, and

iii.) $d(x, z) \leq d(x, y)+d(y, z)$.

To generalize $i$, we need a d.d.f. that corresponds to a distance of zero. Based on the probability interpretation of d.d.f., we need a function that says the properties of the distance being less than or equal to 0 is 1 . This is, in our notation, the function

$$
d_{0,1}(x)= \begin{cases}0 & \text { if } x=0 \\ 1 & \text { if } x>0\end{cases}
$$

The probabilistic version of $i i$ is straightforward, we simply assign the same d.d.f. to the pair $(x, y)$ as to $(y, x)$.

In order to find an analog of the triangle inequality in iii, we need a suitable function from $\Delta^{+} \times \Delta^{+}$into $\Delta^{+}$. These are the triangle functions defined below.

DEFINITION 6.2 (triangle function). We say that $\tau: \Delta^{+} \times \Delta^{+} \rightarrow \Delta^{+}$is a triangle function if $\tau$ assigns a distance distribution function to every pair of distance distribution functions and satisfies the following conditions:

i. $\tau(F, G)=\tau(G, F)$ for all $F, G \in \Delta^{+}$, 
ii. $\tau(F, G) \leq \tau(H, K)$ whenever $F \leq H, G \leq K$ in $\Delta^{+}$,

iii. $\tau\left(F, \delta_{0,1}\right)=F$ for all $F \in \Delta^{+}$,

iv. $\tau(\tau(F, G), H)=\tau(F, \tau(G, H))$ for all $F, G, H \in \Delta^{+}$.

A triangle function is a binary operation on a set of d.d.f.'s (Definition 6.1) that is commutative, associative, and nondecreasing in each place, and has an identity, $\delta_{0,1}$ (Note that Schweizer and Sklar call this $\epsilon_{0}$ ). Now, we are ready for the definition of a PM space.

DEFINITION 6.3 (probabilistic metric space). A probabilistic metric space (briefly, a PM space) is a triple $(S, \mathcal{F}, \tau)$ where $S$ is a nonempty set (whose elements are the points of the space), $\mathcal{F}$ is a function from $S \times S$ into $\Delta^{+}$(the probabilistic metric), $\tau$ is a triangle function, and the following are satisfied for all $p, q, r$ in $S$ :

i. $\mathcal{F}(p, p)=\epsilon_{0}$, for all $p \in S$.

ii. $\mathcal{F}(p, q) \neq \epsilon_{0}$ if $p \neq q \in S$.

iii. $\mathcal{F}(p, q)=\mathcal{F}(q, p)$ for all $p, q \in S$.

iv. $\mathcal{F}(p, r) \geq \tau(\mathcal{F}(p, q), \mathcal{F}(q, r))$ for all $p, q, r, s \in S$.

The other notion that must be discussed is that of contractions, or contraction mappings. First, we provide the well-known definition of a contraction mapping on a general metric space.

DEFINITION 6.4. [contraction mapping, contraction] On a metric space, $M$, with metric, $d$, that is, $(M, d)$, a contraction mapping (or contraction) is a function $f$ from $M$ to itself with the property that there is some nonnegative real number $0 \leq k<1$ such that for all $x, y$ in $M$,

$$
d(f(x), f(y)) \leq k d(x, y) .
$$


Since our goal is to use results from our previous chapter to provide a new definition of construction on a PM space, we start by providing the definition from [11] for contraction mapping on PM spaces.

DEFINITION 6.5 (contraction mapping on a PM space). Let $(S, \mathcal{F}, \tau)$ be a $P M$ space and $\phi$ a function from $S$ into $S$. Then, $\psi$ is a contraction map on $(S, \mathcal{F}, \tau)$ if there is an $\alpha$ in $(0,1)$, the contraction constant of $\psi$, such that

$$
F_{\phi p \phi q}(x) \geq F_{p q}(x / \alpha)
$$

for all $p, q$ in $S$ and all $x$ in the extended positive half-line, $[0, \infty]$; or equivalently, if for all $p, q$ in $S$

$$
F_{\phi p \phi q}(x) \geq F_{p q}(j / \alpha)
$$

where $j$ is the identity function on $[0, \infty]$.

Now that we have the necessary terminology, in the next chapter we will look at some existing work done with contraction mappings on PM spaces and present how it compares to our own. 


\section{CHAPTER 7 \\ CONTRACTIONS ON PROBABILISTIC METRIC SPACES}

Contractions on PM spaces were first studied (and defined) in 1966 by V.M. Sehgal who was working in fixed point theory. Later in the 1970s and 1980s, studies continued with A.T. Bharucha-Reid and T.L. Hicks. Viorel Radu ( [8]) states that there are two known types of contraction mappings in probabilistic metric spaces, one by Sehgal and Bharucha-Reid and one by Hicks. Here we will examine some of their results and compare them with our notion of using way-above and way-below to define a contraction mapping on a PM space $(S, \mathcal{F}, \tau)$.

Since a contraction mapping on a metric space as defined in Definition 6.4 maps any pair of points closer together, we need to achieve this also in the probabilistic sense. This means that two points $(x, y)$ get mapped to $(f(x), f(y))$ and that the probability of certain distances need to be larger at smaller distances. This corresponds to "moving" our d.d.f. "left" and "up", which in turn means the original d.d.f. or $(x, y)$ is way-below the new d.d.f. or $(f(x), f(y))$. This leads to

Definition 7.1. Let $(S, \mathcal{F}, \tau)$ be a PM space. Then $f: S \rightarrow S$ is a probabilistic $\omega$-contraction if there is a $\phi: \Delta^{+} \rightarrow \Delta^{+}$such that $F \ll \phi(F)$ for all $F \in \Delta^{+}$with $\uparrow F \neq \varnothing$ and $\phi\left(F_{p, q}\right) \leq F_{f p, f q}$ for all $p \neq q$ in $S$ and all $F_{p, q}$ in $\mathcal{F}$. We will call $\phi$ the $\omega$-construction factor of $f$. Here, way-below is interpreted in Res $([0, \infty],[0,1])$.

In view of Theorem 5.14, we see that $\phi \in \operatorname{Res}(\operatorname{Res}([0, \infty],[0,1]))$ with $\delta_{a, b} \ll$ $\phi\left(\delta_{a, b}\right)$ for all $a \in(0, \infty)$ and $b \in(0,1)$ are the $\omega$-construction functions that will be most useful. 
In a 1983 paper ([6]), T. L. Hicks stated that in a probabilistic metric space, if there is a metric $d$ on $S$ related to the distribution functions by

$$
d(x, y)<t \text { if and only if } F_{x, y}(t)>1-t, \text { for } t>0
$$

then $d$ is called a compatible metric. He used this to translate conditions to PM spaces, especially the contraction condition. His result is presented in Theorem 7.2.

DEFINITION 7.2 (Hicks 1983). The following condition is a reasonable generalization of a contraction to PM-spaces:

$F: S \rightarrow S$ is a contraction if there is a $k$ such that for $t>0$,

$$
F_{f x, f y}(k t)>1-k t \text { whenever } F_{x, y}(t)>1-t
$$

In order to compare our Definition 7.1 to that of other authors, we need to rephrase theirs in terms of our $\omega$-construction function $\phi$. In particular, if

$$
F_{p, q} \nless F_{f p, f q}
$$

we see that the two notions are not comparable.

THEOREM 7.3. If we have for all $t>0$,

$$
F_{f x, f y}(k t)>1-k t \text { if and only if } F_{x y}(t)>1-t \text {, }
$$

then this neither implies nor is implied by

$$
F_{x, y} \ll F_{f x, f y}
$$

Proof. Let $0<k<1$ be fixed and let $t>\frac{3}{4}$. 
Consider the functions:

$$
\begin{gathered}
G(x)=\delta_{\frac{3}{4}, 1} \\
F(x)=\delta_{\frac{3}{8}, \frac{3}{4}} \\
\text { If } k=\frac{1}{2} \text {, we have } G_{x, y}(t)>1-t . \text { Also, } \\
F\left(\frac{1}{2} t\right)=\frac{3}{4}>1-\frac{1}{2} \cdot \frac{3}{4}=\frac{5}{8} .
\end{gathered}
$$

So, the condition for Hicks' definition is satisfied, however, $G \| F$, in particular, $G \nless F$.

Now consider:

$$
\delta_{\frac{1}{4}, \frac{1}{2}}=G \ll F=\delta_{\frac{1}{8}, \frac{3}{4}}
$$

For $G(t)>1-t$, we must have $t>\frac{1}{4}$. This means,

$$
\begin{aligned}
F(k t)>1-k t \text { if and only if } \frac{3}{4} & >1-\frac{1}{4} k \\
3 & >4-k \\
-1 & >-k \\
k & >1
\end{aligned}
$$

So, given two functions $G \ll F$, does not imply there is a $0<k<1$ which satisfies Hicks' Fixed Point Theorem.

We conclude that the contraction on PM-spaces introduced by T.L. Hicks is not directly related to the way-below relation. 
In 1972, V.M. Sehgal and A.T. Bharucha-Reid published some different work on contraction mappings in probabilistic metric spaces [12]. For simplicity, we will present their result using the notation from a paper written by Schweizer, Sherwood, and Tardiff [10]. They referred to Sehgal and Bharucha-Reid's notion as a B-contraction.

DEFINITION 7.4 (B-contraction). A mapping $f$ of a PM space, $(S, \mathcal{F}, \tau)$ into itself is a B-contraction if there is a $\gamma$ in $(0,1)$ such that for all points $p, q$ in $S$ and all $x>0$,

$$
F_{f p, f q}(\gamma x) \geq F_{p q}(x)
$$

As it turns out, these B-contractions are also not directly related to wayabove. Our notion of way-above is actually stronger in some sense.

In Sehgal and Bharucha-Reid's contraction, we can consider there is a function, $T$ that maps $F_{p, q} \rightarrow F_{f p, f q}$ and that $T(F)(x)=F(\gamma x)$ for some $\gamma$ in $(0,1)$. Since the $(\gamma x)$ gives us a scaling along the $x$-axis and we have a greater than or equal relationship, this $T$ was a potential match for our $\omega$-construction function $\phi$ that maps way-above. However, in some cases, this scaling along the $x$-axis was only enough to "shift left" and not "shift up" as we have required for way-above. So we consider the following example.

EXAMPLE 7.5. Let $F_{p q}=\delta_{\frac{1}{2}, \frac{1}{2}}$ and $\gamma=\frac{1}{2}$. Then, we have

$$
T\left(\delta_{\frac{1}{2}, \frac{1}{2}}\right)=\delta_{\frac{1}{4}, \frac{1}{2}} \geq \delta_{\frac{1}{2}, \frac{1}{2}}
$$

satisfying the idea of B-contraction. However,

$$
\delta_{\frac{1}{4}, \frac{1}{2}} \nless k \delta_{\frac{1}{2}, \frac{1}{2}}
$$

Thus, these B-contractions are not always related to way-above. 
It is relevant to note that there is some overlap. A $\omega$-construction function $\phi$ can lead to a B-contraction. This would be when $\phi: \Delta^{+} \rightarrow \Delta^{+}$is an order automorphism of Type 1 with $\theta(t)>t$ where $t \in(0,1)$ and $\gamma(x)=k x$ for $k<1$ and $x \in(0, \infty)$.

To add to the discussion, we present that our notion of way-above along with the proper $\phi$ is another type of contraction on PM spaces. In a general metric space, a contraction is where the distance between two points diminishes to zero (or where you have a single point) by a factor of $0<k<1$. Where $f$ is the contraction mapping, this is modeled by

$$
d(f(p), f(q)) \leq k \cdot d(p, q)
$$

for all $p, q$ in the space. We note that $k$ does depend on $f$ but works for any two points in the space. Applying the contraction again yields

$$
d\left(f^{2}(p), f^{2}(q)\right) \leq k \cdot d(f(p), f(q)) \leq k^{2} \cdot d(p, q) .
$$

Thus, iteratively applying the contraction $f$ makes the distance smaller by a factor of $k$. We note that $f$ alone can make the distance smaller, and the $k$ guarantees that this distance converges to zero. This is much like the fact that we require both a shift left and up for way-above.

Now, in a probabilistic metric space, the value of the metric is a probability distribution function (or distance distribution function) represented by $F$ as in the Sehgal definition. If we look back at this representation,

$$
F_{p, q} \leq F_{f p, f q},
$$

applying our contraction (a $\phi$ that maps way-above) is actually squeezing in between the left and right side,

$$
F_{p, q} \ll \phi\left(F_{p, q}\right) \leq F_{f p, f q}
$$


As pointed out in the earlier example $\phi\left(F_{p, q}\right)$ could be equal to $F_{f p, f q}$, but only for a select group of d.d.f.'s.

Our contraction, $\phi$, is much like that of the factor $k$. We require that the same $\phi$ work for all d.d.f.'s, $F_{p, q}$, and $\phi$ is way-above the identity map. Continuing to iterate $\phi$ brings us back to the result of Chapter 5 ,

$$
\phi^{n}\left(F_{p, q}\right) \rightarrow \delta_{0,1}
$$

In this context with $\delta_{0,1}$ being a d.d.f., it means the probability that points $p$ and $q$ have a distance of zero or less is 1 , and the probability that they have a distance of more than zero is 0. Effectively, two points with the distance distribution function, $\delta_{0,1}$, have 0 distance. This makes $\phi$ a contraction because in the general metric since, continuing to apply $\phi$ makes the distance between two points diminish to a single point. In the future, we plan to investigate further and consider fixed points in PM spaces. 


\section{CHAPTER 8 REMARKS AND CONCLUSION}

This dissertation consists of three parts: understanding posets and basic theory of lattices, working with the way-below relation and how it extends to function spaces and mappings between them, and the basic understanding of probabilistic metric spaces and where the notion of way-below was used to provide an alternative definition of contraction.

After spending time studying special posets and what makes them a lattice, it was particularly interesting to work on problems where the elements of these sets were functions. It also became necessary to examine the behavior of these sets when mapping them back into themselves or other lattices. In this dissertation, we began our work with residuted maps and made progress in generalizing to functions with less restriction.

The basic notion of residuated maps were being studied in the early 1960's by G.N. Raney when he began to look at Galois connections on lattices. Later, Blyth and Janowitz brought residuated maps to the forefront in this 1972 book [1]. There are still unanswered questions about the residuated maps particular lattices induce and how the relationship between certain lattices affect or provide the relationship between their associated sets of residuated maps. This leaves avenues for future research in the area and even exploratory work on an undergraduate project.

The way-below relation was studied on some well-known but restrictive spaces of residuated maps.A characterization of when $G \ll F$ for $F, G \in \operatorname{Res}(L, M)$ where $L, M$ are more general lattices than the chains $[p, q],[r, s]$ is one of the fu- 
ture plans together with extending other results from this dissertation to this more general setting. It is an example of some notions we encounter where the formal definition, the graphical interpretation, and using it in practice are not necessarily the same in appearance. This work provided a carefully examined characterization of functions that have the way-below relationship. Through examples and proof, we were able to determine criteria necessary for excluding functions from having another function way-above and when this relationship does exist under which mappings is it preserved.

The culmination of these efforts was that the iteration of a function $\phi$ that always maps functions way-above and sends deltas to deltas yields the top element of the lattice, or in our case $\delta_{0,1}$. Throughout the time spent on way-below, we continually changed restrictions on $\phi$ and made much headway on generalizing. While we were able to provide sufficient conditions for mappings on $\operatorname{Res}([p, q],[r, s])$ that guarantee $\phi^{n}(F) \rightarrow \delta_{0,1}$, future work will need to look at what conditions are necessary. We did provide one example of a monotone function, which is not residuated and does not iterate to $\delta_{0,1}$, so we have paved the way in that direction.

The last part of our study was making connections between the way-below relation and probabilistic metric spaces. By looking at some current definitions of contractions, we were able to make the case that our $\phi$ that maps way-above provides another definition for contraction. This too opens new doors for potential study. We intend to continue looking at our contraction and what conditions if any can be relaxed as well as begin a study on fixed points in PM spaces and their uniqueness. 


\section{REFERENCES}

[1] T.S. Blyth and M.F. Janowitz, Residuation Theory, Pergamon Press, Oxford, 1972.

[2] G. L. Cain and R. H. Kasriel, Fixed and periodic points of local contraction mappings on probabilistic metric spaces, Mathematical systems theory 9 (1975), no. 3, 289-297.

[3] B.A. Davey and H.A. Priestley, Introduction to Lattices and Order, 2 ed., Cambridge University Press, Cambridge, 2002.

[4] Michael Edelstein, An extension of banach's contraction principle, Proceedings of the American Mathematical Society 12 (1961), no. 1, 7-10.

[5] Gerhard Gierz, Karl Heinrich Hofmann, Klaus Keimel, Jimmie D. Lawson, Michael W. Mislove, and Dana S. Scott, A compendium of continuous lattices, Springer-Verlag, Berlin-New York, 1980. MR 614752

[6] T.L. Hicks, Fixed point theory in probabilistic metric spaces, Univ. u Novom Sadu Zb. Rad. Prirod.-Mat. Fak. Ser. Mat. 13 (1983), 63-72.

[7] Robert C Powers, Order automorphisms of spaces of nondecreasing functions, Journal of Mathematical Analysis and Applications 136 (1988), no. 1, 112 123.

[8] Viorel Radu, Some fixed point theorems probabilistic metric spaces, pp. 125133, Springer Berlin Heidelberg, Berlin, Heidelberg, 1987. 
[9] B.E. Rhoades, A comparison of various definitions of contractive mappings, Transactions of the American Mathematical Society (1977), 257-290.

[10] B. Schweizer, H. Sherwood, and R.M. Tardiff, Contractions on probabilistic metric spaces: examples and counterexamples, Stochastica 13 (1988), no. 1, $5-17$.

[11] B. Schweizer and A. Sklar, Probabilistic metric spaces, Dover Publications, Inc., Mineola, NY, 2005.

[12] V. M. Sehgal and A. T. Bharucha-Reid, Fixed points of contraction mappings on probabilistic metric spaces, Mathematical systems theory 6 (1972), no. 1, $97-102$. 
CURRICULUM VITAE

Michael Ryan Luke

EDUCATION

University of Louisville, Louisville, KY

Ph.D. Applied \& Industrial Mathematics

August 2017

University of Louisville, Louisville, $K Y$

M.A. Mathematics

December 2013

Mississippi College, Clinton, MS

M.S. Mathematics

December 2011

Graduated Summa Cum Laude

University of Mississippi, Oxford, MS

TEACH Mississippi Institute Teacher Pre-Licensing Certificate 2010

Mississippi College, Clinton, MS

B.S. Computer Science

May 2002

Minor in Mathematics 


\section{TEACHING EXPERIENCE}

\section{University of Louisville, Louisville $K Y$}

\section{Graduate Teaching Assistant in Mathematics}

Primary Instructor for the following courses:

- MATH 151: Mathematics for Elementary Education I

Prepared future elementary educators for teaching problem solving skills and mathematical reasoning. This is a required lecture and lab for admittance to the Elementary Teacher Program which focuses on problem solving, number systems, number theory, and operations. Also utilized a basic skills assessment to ensure candidate fluency.

- MATH 152: Mathematics for Elementary Education II

This is a required lecture and lab continuation of Mathematics for Elementary Education I and features elements of geometry, patterns, statistics, probability, and counting. This is a required preparation class for admittance to the Elementary Education program.

- MATH 109: Elementary Statistics

A required statistics course for Nursing majors, some education disciplines, and other programs with a possible research element in which data collection, trials, ethics, descriptive statistics, normal and binomial distributions, $t$ and $\chi^{2}$ tests, inferential statistics, contingency tables, correlation, and regression are all discussed.

- MATH 111: College Algebra

Assisted students with mastering the ability to solve equations and in- 
equalities involving rational, exponential and logarithmic equations while using them to build logical thinking and problem solving skills.

- MATH 111S: Supplemented College Algebra Helped students who were possibly struggling in Math to develop critical thinking skills and algebraic skills that are required in general education and higher mathematics, especially those traditionally needed to receive credit for College Algebra

- MATH 190: Pre-Calculus in Summer 2014

Worked with students to recall and learn techniques from Algebra and Trigonometry which are necessary for success in the traditional Calculus sequence. Presented questions and problem-solving ideas in order to polish these skills and foster critical thinking.

- MATH 205: Calculus 1 in Spring 2014 \& Spring 2016

Unveiled the remarkable set of tools in differential and integral calculus of single variable functions to students seeking degrees in natural and applied sciences. This course is required for STEM field majors.

- MATH 180: Elements of Calculus in Summer 2012 and Summer 2013 A calculus class required for business majors and majors in the life sciences where I helped students from multiple disciplines see how the tools of Calculus including limits, derivatives, and integrals could enable them make sound business decisions, analyze economic data or reports, and interpret scientific observations.

Teaching Assistant for the following courses:

- MATH 105: Contemporary Mathematics

- MATH 180: Elements of Calculus

- MATH 109: Elementary Statistics 
- MATH 111: College Algebra

Mississippi College, Clinton, MS

Graduate Teaching Assistant II \& Adjunct Instructor in Mathematics 2011

Primary Instructor for the following course:

- MATH 111: College Algebra

Helped students to develop critical thinking skills and algebraic skills that are required in general education and higher mathematics; Assisted students with mastering the ability to solve equations and inequalities involving rational, exponential and logarithmic equations while using them to build logical thinking and problem solving skills.

Teaching Assistant for the following courses:

- MATH 101: College Algebra

- MATH 121: Calculus I

- MATH 122: Calculus II

Clinton, $M S$

Math Tutor

Worked with local students that needed additional instruction or help with math outside class 
AWARDS

- Dean's Citation - Master's Degree, University of Louisville

- Spirit of Student Government Award

2015

- Dean's Dedicated SGA Senator Award

2016

- Induction to Pi Mu Epsilon (Math Honor Society)

2011

- President's List, Mississippi College

- Graduate School Dean's List, Mississippi College

2011

- Dean's List, Mississippi College

2000-2001

- Dean's List, Mississippi State University

1999

\section{LEADERSHIP}

Student Government Association, University of Louisville

Graduate Student Senator

$2014-2016$

Graduate Student Executive Council, University of Louisville

Director of Travel

$2014-2016$

Representative

Spring 2014 - Summer 2016 
Graduate Student Union, University of Louisville

President

Spring 2015-2016

Vice President

Spring 2014 - Spring 2015

Representative

Spring 2014 - 2016

Graduate School, University of Louisville

Graduate Student Ambassador

2014-2017

\section{TRAINING}

- Graduate Teaching Assisstant Academy 2014-2015

- CITI Research Training \& IRB Approval

- GTA's and Diversity PLAN Series: Understanding Diversity in Academia

- regular participation in PLAN Events: such as Online Teaching, Time Management, Classroom Assessment Techniques, Grant Writing, Publishing, etc.

MENTORING / SERVICE

- Personally Managed $\$ 74,000$ in travel funding for Graduate Students as Travel Administrator for GSC. Continually reviewed, approved, and processed funding for over 600 students.

- Selected recipients and appropriated over $\$ 37,000$ in research grants. 
- Served on multiple selection committees which read and deliberated ultimately distributing funding for about 50 Graduate Students in Arts \& Sciences.

- Work on a faculty committee (as a graduate student) to develop \& plan the best preparation and course experience for future educators.

- Regular participation in joint planning, problem solving, and brainstorming sessions with College of Education and Department of Mathematics

- Assisted with organization for the American Mathematical Society Sectional Meeting being held at the University of Louisville Fall 2013

- Peer Mentor for Mathematics Graduate Students

- Maintain a Bulletin Board for Graduate Students in the Mathematics Department

- Participate in Graduate Student Council and Graduate Student Union Meetings and Events

- Email and Post announcements about events and meetings involving graduate students and spread news regarding the university and student body

\section{PUBLICATIONS}

- Luke, Ryan. (2014). "Using the XYZ Homework System" In Marti Zimmerman, College algebra: a problem-solving approach (pp. iv - viii) Cincinnati, OH: Van-Griner, LLC. 


\section{PRESENTATIONS}

- "The Way-Below Relation and Maps on $\operatorname{Res}(L, M)$ ", Mathematics Association of America Sectional Meeting, Berea College, Berea, KY, March 2017

- "Research with the Way-Below Relation on Residuated Maps", Candidacy Exam, University of Louisville, Kentucky, October 2016

- "Residuated Maps and the Way-Below Relation", Mathematics Association of America Sectional Meeting, Highland Heights, Kentucky, April 2016

- "Observations and Experiences with the Common Core Standards for Mathematical Practice", Mathematics Association of America Sectional Meeting, Moorehead, Kentucky, April 2015

- "Finding groups among residuated mappings on a lattice L", Mathematics Association of America Sectional Meeting, Murray, Kentucky, April 2014

- "Maximum Modulus Principle", University of Louisville, Louisville, Kentucky, April 2013

\section{CONFERENCE ATTENDANCE}

- Mathematics Association of America Sectional Meeting, Northern Kentucky University, Highland Heights, KY, April 2016

- Mathematics Association of America Sectional Meeting, Moorehead State University, Moorehead, KY, April 2015 
- Developmental Education Institute, Kentucky Council on Post-Secondary Education, Louisville, KY, October 2014

- Mathematics Association of America Sectional Meeting, Murray State University, Murray, KY, April 2014

- Mathematics Association of America Sectional Meeting, Transylvania University, Lexington, Kentucky, April 2013

- Annual Mathematics Symposium, Western Kentucky University, Bowling Green, Kentucky, September 2012

- Mathematics Association of America Sectional Meeting, Bellarmine University, Louisville, Kentucky, April 2012

- National Joint Mathematics Meeting, Boston, MA, January 2012

\section{AFFILIATIONS}

- Mathematics Association of America

- American Mathematics Society

- Pi Mu Epsilon Math Honor Society

- Association of Christians in the Mathematical Sciences

- invited to join Golden Key Honor Society

- invited to join Omicron Delta Kappa 